\title{
Theoretical aspects of mixed volume computation via mixed subdivision
}

\author{
Tianran Chen*, Tien-Yien Li* and XiaOshen Wang
}

\begin{abstract}
We analyze the computation of mixed volume of tuples of polytopes via fine mixed subdivisions. This method expresses the mixed volume as a sum of easily computable standard volumes of polytopes called mixed cells. Mixed cells play a critically important role in polyhedral homotopy continuation methods, which in turn are a particularly efficient numerical method for the solution of systems of polynomial equations. We provide a complete and selfcontained account of the underlying computational convexity techniques, assuming no background in algebraic geometry.
\end{abstract}

\section{Introduction}

Throughout this article, $n$ represents an arbitrary but fixed positive integer. For a set $A \subset \mathbb{R}^{m}$, we denote its closure, interior, boundary and convex hull by $\bar{A}$, int $A, \partial A$ and conv $A$ respectively. For two sets $A, B \subset \mathbb{R}^{m}, A+B$ denotes their Minkowski sum [25] $\{\boldsymbol{a}+\boldsymbol{b}: \boldsymbol{a} \in A$ and $\boldsymbol{b} \in B\} \subset \mathbb{R}^{m}$. With a positive real number $\lambda$ and a set $A \subset \mathbb{R}^{m}$, the scaling of $A$ by the factor $\lambda$ is simply the result of applying the scaling to each point of $A$, that is, $\lambda A=\{\lambda \boldsymbol{a}: \boldsymbol{a} \in A\}$. Furthermore, $\operatorname{Vol}_{n} A$ is the standard volume of $\mathbb{R}^{n}$, and $\langle\cdot, \cdot\rangle$ stands for the standard Euclidean inner product on $\mathbb{R}^{n}$.

For $i=1, \ldots, n$, let $\mathcal{S}_{i}$ be a finite subset of $\mathbb{R}^{n}$ and $\mathcal{Q}_{i}=\operatorname{conv} \mathcal{S}_{i}$. Our interest will be focused on the $n$-tuple of sets $\mathcal{S}=\left(\mathcal{S}_{1}, \ldots, \mathcal{S}_{n}\right)$ and the $n$-tuple of convex polytopes $\mathcal{Q}=\left(\mathcal{Q}_{1}, \ldots, \mathcal{Q}_{n}\right)$. For positive real numbers $\lambda_{1}, \ldots, \lambda_{n}$, the $n$-dimensional volume of the Minkowski sum

$$
\lambda_{1} \mathcal{Q}_{1}+\cdots+\lambda_{n} \mathcal{Q}_{n}=\left\{\lambda_{1} \boldsymbol{q}_{1}+\cdots+\lambda_{n} \boldsymbol{q}_{n}: \boldsymbol{q}_{i} \in \mathcal{Q}_{i} \text { for } i=1, \ldots, n\right\}
$$

is a homogeneous polynomial of degree $n$ in the variables $\lambda_{1}, \ldots, \lambda_{n}$. We call the coefficient of the monomial $\lambda_{1} \times \cdots \times \lambda_{n}$ in this polynomial the mixed volume of $\left(\mathcal{Q}_{1}, \ldots, \mathcal{Q}_{n}\right)[10,13,25]$, denoted by $\mathcal{M}\left(\mathcal{Q}_{1}, \ldots, \mathcal{Q}_{n}\right)$,

\footnotetext{
* Research supported in part by NSF under Grant DMS 11-15587.
} 
or simply $\mathcal{M}(\mathcal{Q})$. The mixed volume of convex polytopes is a classical concept in combinatorial geometry, dating back to works of Minkowski in the early 1900s. Mixed volume, thanks to its connections to Hodge theory and toric varieties (see, e.g., [6, 18, 28]), is an important invariant from convex geometry that has since proved important in numerous applications related to solving polynomial systems.

Among various approaches to computing the mixed volume $\mathcal{M}(\mathcal{Q})$ (such as [3, 7, 9, 24]), we will detail fine mixed subdivisions of the $n$-tuple $\mathcal{S}=$ $\left(\mathcal{S}_{1}, \ldots, \mathcal{S}_{n}\right)$ introduced in [16]. This method produces as a by-product the mixed cells which play a critically important role in the construction of efficient polyhedral homotopy continuation methods [5, 15, 16, 20, 21, 29] in solving polynomial systems numerically. Moreover, fine mixed subdivisions are also constructions of great interest in the context of tropical algebraic geometry [17] because they help represent intersections of tropical hypersurfaces 14 .

This article is organized as follows: In 92 , the concept of fine mixed subdivisions is introduced, followed by an exploration of a very important property of the fine mixed subdivision, its scaling invariance, in $\$ 3$. We then detail the concrete method for computing mixed volume via a (fine) mixed subdivision in $\$ 4$. In $\$ 5$ a method for finding a (fine) mixed subdivision via generic lifting is discussed which also constitutes a constructive proof to the existence of a (fine) mixed subdivision. This method reduces the problem of mixed volume computation into a computation to list all mixed cells in a mixed subdivision. Special cases where some of polytopes in $\mathcal{Q}=$ $\left(\mathcal{Q}_{1}, \ldots, \mathcal{Q}_{n}\right)$ are identical, known as semi-mixed cases, are studied in $\$ 6$. It indicates that the computation procedure for computing mixed volume can be modified to take advantage of these special structures. We conclude the article in $\S 7$ with a brief discussion of the concrete formulation of the mixed cell enumeration problem in terms of linear programming problems.

\section{Fine mixed subdivisions}

Recall that a convex polytope $P \subset \mathbb{R}^{n}$ is the convex hull of finite many points in $\mathbb{R}^{n}$. Since all polytopes considered in this article are convex, they will therefore simply be known as polytopes. A subset $F$ of polytope $P$ is called a face of $P$ if there exists $\boldsymbol{\alpha} \in \mathbb{R}^{n}$ for which the linear functional $f(\boldsymbol{x})=\langle\boldsymbol{\alpha}, \boldsymbol{x}\rangle$ on $\mathbb{R}^{n}$ attains its minimum over $P$ at $F$. In this case, the vector $\boldsymbol{\alpha}$ is called an inner normal of $F$. When $P$ is an $n$-dimensional polytope in $\mathbb{R}^{n}$, its $(n-1)$-dimensional faces are called facets of $P$. A subdivision of a polytope $\widetilde{\mathcal{Q}}$ is a collection of polytopes intersecting only along their common faces 
and whose union is the entire $\widetilde{\mathcal{Q}}$. As a point of departure, we generalize this concept to the context of an $n$-tuple of polytopes.

Definition 1 (Subdivision). A subdivision of $\mathcal{S}=\left(\mathcal{S}_{1}, \ldots, \mathcal{S}_{n}\right)$ is a collection $\mathcal{D}$ of $n$-tuples of the form $C=\left(C_{1}, \ldots, C_{n}\right)$, called cells, with $\varnothing \neq$ $C_{i} \subseteq \mathcal{S}_{i}$ for each $i=1, \ldots, n$ such that

(a): $\operatorname{dim}\left(\operatorname{conv} C_{1}+\cdots+\operatorname{conv} C_{n}\right)=n$ for all $C \in \mathcal{D}$,

(b): For a distinct pair $A, B \in \mathcal{D}$, if $(\operatorname{conv} A) \cap(\operatorname{conv} B)$ is nonempty, then it is a common face of both,

(c): $\bigcup_{\left(C_{1}, \ldots, C_{n}\right) \in \mathcal{D}}\left(\operatorname{conv} C_{1}+\cdots+\operatorname{conv} C_{n}\right)=\operatorname{conv} \mathcal{S}$.

For each cell $C=\left(C_{1}, \ldots, C_{n}\right) \in \mathcal{D}$, we use the notations

$$
\begin{aligned}
\text { type } C & =\left(\operatorname{dim}\left(\operatorname{conv} C_{1}\right), \ldots, \operatorname{dim}\left(\operatorname{conv} C_{n}\right)\right) \\
\operatorname{conv} C & =\operatorname{conv} C_{1}+\cdots+\operatorname{conv} C_{n} \\
\operatorname{Vol}_{n} C & =\operatorname{Vol}_{n}(\operatorname{conv} C)
\end{aligned}
$$

Note that the above conditions describe a subdivision of the single polytope $\widetilde{\mathcal{Q}}=\mathcal{Q}_{1}+\cdots+\mathcal{Q}_{n}$ with $\mathcal{Q}_{i}=\operatorname{conv} \mathcal{S}_{i}$ for $i=1, \ldots, n$, with the only special restriction that each cell in the subdivision must be the Minkowski sum of $n$ polytopes whose vertices lie in $\mathcal{S}_{1}, \ldots, \mathcal{S}_{n}$ respectively.

While a subdivision of $\widetilde{\mathcal{Q}}$ is important in computing the volume of $\widetilde{\mathcal{Q}}$ (as long as the volume of each sub-polytope is easy to obtain), in studying mixed volume computation, it is more important to find the expression of the volume of the Minkowski sum $\lambda_{1} \mathcal{Q}_{1}+\cdots+\lambda_{n} \mathcal{Q}_{n}$ in relation to the factors $\lambda_{1}, \ldots, \lambda_{n}$. For this purpose, a mere subdivision is insufficient. Explicitly, for $\boldsymbol{\lambda}=\left(\lambda_{1}, \ldots, \lambda_{n}\right) \in\left(\mathbb{R}^{+}\right)^{n}$, we use the notation $\boldsymbol{\lambda} \circ \mathcal{S}$ for the scaled version $\left(\lambda_{1} \mathcal{S}_{1}, \ldots, \lambda_{n} \mathcal{S}_{n}\right)$. Similarly, for a cell $C=\left(C_{1}, \ldots, C_{n}\right)$ in a subdivision $\mathcal{D}$ of $\mathcal{S}=\left(\mathcal{S}_{1}, \ldots, \mathcal{S}_{n}\right)$, we used the notation $\lambda \circ\left(C_{1}, \ldots, C_{n}\right)=\left(\lambda_{1} C_{1}, \ldots, \lambda_{n} C_{n}\right)$. An example below will illustrate that a subdivision $\mathcal{D}$ of $\mathcal{S}=\left(\mathcal{S}_{1}, \ldots, \mathcal{S}_{n}\right)$ does not, in general, extend properly under such "mixed" scaling operation. Namely,

$$
\boldsymbol{\lambda} \circ \mathcal{D}:=\left\{\boldsymbol{\lambda} \circ C=\left(\lambda_{1} C_{1}, \ldots, \lambda_{n} C_{n}\right) \mid C=\left(C_{1}, \ldots, C_{n}\right) \in \mathcal{D}\right\}
$$

may not be a subdivision of $\boldsymbol{\lambda} \circ \mathcal{S}$. Inevitably, to make a subdivision scaling invariant, which turns out to be a critically important property for our mixed volume computation, additional restrictions are necessary: 
Definition 2 (Mixed subdivision). A subdivision $\mathcal{D}$ of $\mathcal{S}=\left(\mathcal{S}_{1}, \ldots, \mathcal{S}_{n}\right)$ is called a mixed subdivision if, in addition, it satisfies

(d1): For each cell $C=\left(C_{1}, \ldots, C_{n}\right) \in \mathcal{D}, \sum_{i=1}^{n} \operatorname{dim}\left(\operatorname{conv} C_{i}\right)=n$,

(d2): For any distinct pair of cells $A=\left(A_{1}, \ldots, A_{n}\right)$ and $B=\left(B_{1}, \ldots\right.$, $\left.B_{n}\right)$ in $\mathcal{D}$,

$$
(\operatorname{conv} A) \cap(\operatorname{conv} B)=\sum_{i=1}^{n}\left(\operatorname{conv} A_{i} \cap \operatorname{conv} B_{i}\right) .
$$

Cells of a mixed subdivision are called mixed cells.

As Example 5 below shows, these two conditions are essential in relating the cells in a subdivision and the volume $\operatorname{Vol}_{n}\left(\lambda_{1} \mathcal{Q}_{1}+\cdots+\lambda_{n} \mathcal{Q}_{n}\right)$ by which the mixed volume is defined.

A mixed subdivision of $\mathcal{S}=\left(\mathcal{S}_{1}, \ldots, \mathcal{S}_{n}\right)$ may be refined via further subdivision of individual components of each cell. It is computationally advantageous (as Equation (10) will show later) to consider the most refined mixed subdivision:

Definition 3 (Fine mixed subdivision). A mixed subdivision $\mathcal{D}$ of $\mathcal{S}=\left(\mathcal{S}_{1}, \ldots, \mathcal{S}_{n}\right)$ is called a fine mixed subdivision if it also satisfies the condition

(e): For each cell $C=\left(C_{1}, \ldots, C_{n}\right) \in \mathcal{D}$, conv $C_{i}$ is a simplex of dimension $\# C_{i}-1$ for $i=1, \ldots, n$.

For mixed subdivisions (and fine mixed subdivisions), we then have the important property of scaling invariance:

Proposition 4. Let $\mathcal{D}$ be a (fine) mixed subdivision of $\mathcal{S}=\left(\mathcal{S}_{1}, \ldots, \mathcal{S}_{n}\right)$. Then for any $\boldsymbol{\lambda}=\left(\lambda_{1}, \ldots, \lambda_{n}\right) \in\left(\mathbb{R}^{+}\right)^{n}$, the set

$$
\boldsymbol{\lambda} \circ \mathcal{D}:=\left\{\boldsymbol{\lambda} \circ C=\left(\lambda_{1} C_{1}, \ldots, \lambda_{n} C_{n}\right) \mid C=\left(C_{1}, \ldots, C_{n}\right) \in \mathcal{D}\right\}
$$

forms a (fine) mixed subdivision of $\boldsymbol{\lambda} \circ \mathcal{S}=\left(\lambda_{1} \mathcal{S}_{1}, \ldots, \lambda_{n} \mathcal{S}_{n}\right)$.

This proposition lays the ground for the approach of mixed volume computation we shall discuss in this article. The complete proof of it is somewhat technical, it will be detailed in the next section. In the following example, we shall illustrate the importance of the condition (d2). Without it, the subtlety of nice scaling behavior of the subdivision may disappear. 
Example 5 (Subdivision without condition (d2)). With $n=2$, consider two finite subsets $\mathcal{S}_{1}=\{(0,0),(1,0),(0,1)\}$ and $\mathcal{S}_{2}=\{(0,0),(1,0)$, $(0,1)\}$ in $\mathbb{R}^{2}$ and their convex hulls $\mathcal{Q}_{1}=$ conv $\mathcal{S}_{1}$ and $\mathcal{Q}_{2}=$ conv $\mathcal{S}_{2}$ as shown below
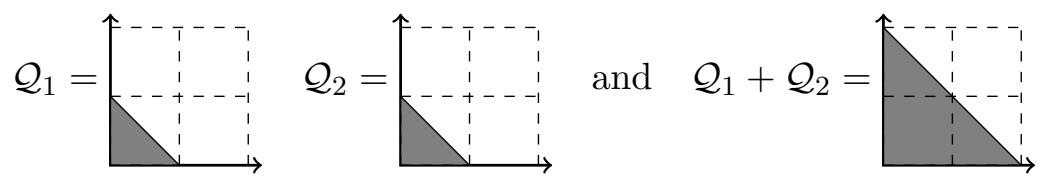

Let $A=(\{(0,0),(1,0),(0,1)\},\{(0,0)\}), B=(\{(0,0),(1,0),(0,1)\},\{(0,1)\})$, and $C=(\{(1,0),(0,1)\},\{(0,0),(1,0)\})$ be three cells of $\mathcal{S}=\left(\mathcal{S}_{1}, \mathcal{S}_{2}\right)$. Let $\mathcal{D}=\{A, B, C\}$. The convex hull of each cell becomes:
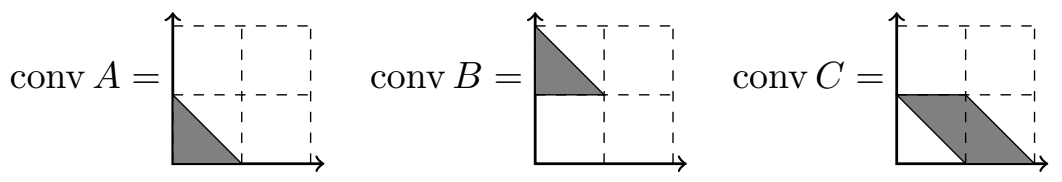

Clearly, $\operatorname{dim}(\operatorname{conv} A)=\operatorname{dim}(\operatorname{conv} B)=\operatorname{dim}(\operatorname{conv} C)=2$ and the intersection of any two is their common face. Furthermore, the union of the three convex hulls

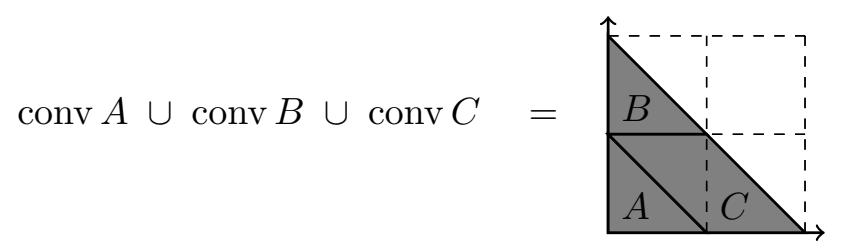

is indeed the entire $\widetilde{\mathcal{Q}}=\mathcal{Q}_{1}+\mathcal{Q}_{2}$. Therefore $\mathcal{D}=\{A, B, C\}$ satisfies the definition of a subdivision (conditions (a),(b),(c)). In fact, since the cells $A, B$, and $C$ are of types $(2,0),(2,0)$, and $(1,1)$ respectively, $\mathcal{D}$ also satisfies condition (d1). But, the failure of condition (d2) for subdivision $\mathcal{D}$ can be easily verified. As a consequence, it may not behave favorably under the mixed scaling by $\boldsymbol{\lambda}=\left(\lambda_{1}, \lambda_{2}\right)$. Namely, for certain scaling $\boldsymbol{\lambda}=\left(\lambda_{1}, \lambda_{2}\right), \boldsymbol{\lambda} \circ \mathcal{D}=$ $(\boldsymbol{\lambda} \circ A, \boldsymbol{\lambda} \circ B, \boldsymbol{\lambda} \circ C)$ may not be a subdivision of $\boldsymbol{\lambda} \circ \mathcal{S}=\left(\lambda_{1} S_{1}, \lambda_{2} S_{2}\right)$. The cells will both separate and overlap as different scaling factors are chosen.

For example, with factors $\boldsymbol{\lambda}=\left(\lambda_{1}, \lambda_{2}\right)=(1,2)$, the scaled version of $\widetilde{\mathcal{Q}}=$ $\mathcal{Q}_{1}+\mathcal{Q}_{2}$ is $\boldsymbol{\lambda} \circ \widetilde{\mathcal{Q}}=1 \cdot \mathcal{Q}_{1}+2 \cdot \mathcal{Q}_{2}:$

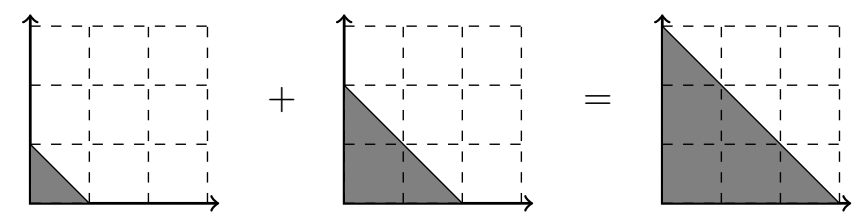


But under the same scaling factors the cells separate with the union of their convex hulls equal to

$$
\operatorname{conv}((1,2) \circ A) \cup \operatorname{conv}((1,2) \circ B) \cup \operatorname{conv}((1,2) \circ C)=\left[\begin{array}{ccc}
1 \\
\vdots \\
\vdots
\end{array}\right] .
$$

It no longer covers the entire $\boldsymbol{\lambda} \circ \widetilde{\mathcal{Q}}=1 \cdot \mathcal{Q}_{1}+2 \cdot \mathcal{Q}_{2}$.

Alternatively, with the scaling factor $\boldsymbol{\lambda}=(2,1), \boldsymbol{\lambda} \circ \widetilde{\mathcal{Q}}=2 \cdot \mathcal{Q}_{1}+1 \cdot \mathcal{Q}_{2}$ becomes

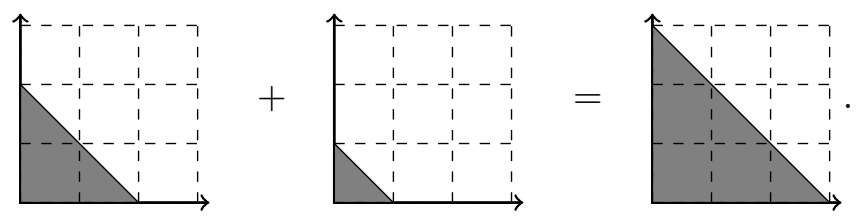

Under the same scaling, $\operatorname{conv}((2,1) \circ A), \operatorname{conv}((2,1) \circ B), \operatorname{conv}((2,1) \circ C)$ become
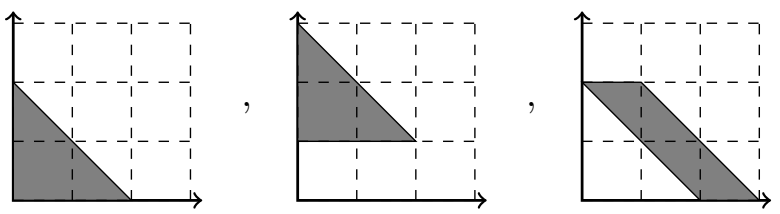

respectively. Clearly there are overlaps among the three.

In both cases, the scaled subdivision $\boldsymbol{\lambda} \circ \mathcal{D}$ failed to form a subdivision of $\boldsymbol{\lambda} \circ \widetilde{\mathcal{Q}}=\lambda_{1} \mathcal{Q}_{1}+\lambda_{2} \mathcal{Q}_{2}$. The problem lies in the fact that the subdivision $\mathcal{D}$ of $\mathcal{S}$ does not satisfy the condition (d2).

Remark 6. The condition (d2) was absent when the "mixed subdivision" was originally defined in [16]. It first appeared in [6].

\section{Scaling invariance of mixed subdivisions}

In this section we will provide a detailed proof of Proposition 4 presented in the last section, that is: If $\mathcal{D}$ is a (fine) mixed subdivision of $\mathcal{S}=$ $\left(\mathcal{S}_{1}, \ldots, \mathcal{S}_{n}\right)$, then for $\boldsymbol{\lambda}=\left(\lambda_{1}, \ldots, \lambda_{n}\right) \in\left(\mathbb{R}^{+}\right)^{n}$,

$$
\boldsymbol{\lambda} \circ \mathcal{D}:=\left\{\boldsymbol{\lambda} \circ C:=\left(\lambda_{1} C_{1}, \ldots, \lambda_{n} C_{n}\right): C=\left(C_{1}, \ldots, C_{n}\right) \in \mathcal{D}\right\}
$$

is a (fine) mixed subdivision of $\boldsymbol{\lambda} \circ \mathcal{S}=\left(\lambda_{1} \mathcal{S}_{1}, \ldots, \lambda_{n} \mathcal{S}_{n}\right)$. 
In the first place, we define the support function: For a nonzero vector $\boldsymbol{\alpha} \in \mathbb{R}^{n}$ and a compact set $P \subset \mathbb{R}^{n}$ define

$$
\mathrm{ht}_{\boldsymbol{\alpha}} P:=\min _{\boldsymbol{p} \in P}\langle\boldsymbol{p}, \boldsymbol{\alpha}\rangle, \quad(P)_{\boldsymbol{\alpha}}:=\left\{\boldsymbol{p} \in P:\langle\boldsymbol{p}, \boldsymbol{\alpha}\rangle=\mathrm{ht}_{\boldsymbol{\alpha}}(P)\right\}
$$

Here the notation "ht $\boldsymbol{\alpha}$ " is used since this function measures the "height" of the set $P$ in the direction given by $\boldsymbol{\alpha}$. It is clear that for $\lambda_{i} \in \mathbb{R}^{+}$and compact sets $P_{i} \subset \mathbb{R}^{n}$ for $i=1, \ldots, n$,

$$
\begin{aligned}
\operatorname{ht}_{\boldsymbol{\alpha}}\left(\lambda_{1} P_{1}+\cdots+\lambda_{n} P_{n}\right) & =\lambda_{1} \mathrm{ht}_{\boldsymbol{\alpha}} P_{1}+\cdots+\lambda_{n} \mathrm{ht}_{\boldsymbol{\alpha}} P_{n} \\
\left(\lambda_{1} P_{1}+\cdots+\lambda_{n} P_{n}\right)_{\boldsymbol{\alpha}} & =\lambda_{1}\left(P_{1}\right)_{\boldsymbol{\alpha}}+\cdots+\lambda_{n}\left(P_{n}\right)_{\boldsymbol{\alpha}} .
\end{aligned}
$$

Furthermore, for compact sets $A, B \subseteq \mathbb{R}^{n}$,

$$
\operatorname{ht}_{\boldsymbol{\alpha}}(A \cup B)=\min \left\{\operatorname{ht}_{\boldsymbol{\alpha}}(A), \operatorname{ht}_{\boldsymbol{\alpha}}(B)\right\},
$$

and hence

$$
(A \cup B)_{\boldsymbol{\alpha}} \subseteq(A)_{\boldsymbol{\alpha}} \cup(B)_{\boldsymbol{\alpha}}
$$

For a finite set $A \subset \mathbb{R}^{n}$,

$$
(\operatorname{conv} A)_{\boldsymbol{\alpha}}=\operatorname{conv}(A)_{\boldsymbol{\alpha}}
$$

For the rest of this section, let $\mathcal{D}$ be a mixed subdivision of $\mathcal{S}=\left(\mathcal{S}_{1}, \ldots\right.$, $\left.\mathcal{S}_{n}\right)$ and $\boldsymbol{\lambda}=\left(\lambda_{1}, \ldots, \lambda_{n}\right)$ be positive scaling factors. We now explore the behavior of a mixed subdivision under scaling via the following lemmas.

Lemma 7. For any cell $C=\left(C_{1}, \ldots, C_{n}\right) \in \mathcal{D}$, if $\boldsymbol{x} \in \operatorname{conv}(\boldsymbol{\lambda} \circ C)$ then there exists a unique n-tuple $\left(\boldsymbol{x}_{1}, \ldots, \boldsymbol{x}_{n}\right)$ with $\boldsymbol{x}_{i} \in C_{i}$ for all $i$ such that $\boldsymbol{x}=\lambda_{1} \boldsymbol{x}_{1}+\cdots+\lambda_{n} \boldsymbol{x}_{n}$.

Proof. Suppose there are pairs $\boldsymbol{x}_{1}, \boldsymbol{x}_{1}^{\prime} \in \operatorname{conv} C_{1}, \ldots, \boldsymbol{x}_{n}, \boldsymbol{x}_{n}^{\prime} \in \operatorname{conv} C_{n}$ such that

$$
\boldsymbol{x}=\lambda_{1} \boldsymbol{x}_{1}+\cdots+\lambda_{n} \boldsymbol{x}_{n}=\lambda_{1} \boldsymbol{x}_{1}^{\prime}+\cdots+\lambda_{n} \boldsymbol{x}_{n}^{\prime}
$$

Then $\lambda_{1}\left(\boldsymbol{x}_{1}^{\prime}-\boldsymbol{x}_{1}\right)+\cdots+\lambda_{n}\left(\boldsymbol{x}_{n}^{\prime}-\boldsymbol{x}_{n}\right)=\mathbf{0}$. For each $i=1, \ldots, n$, let $L_{i}=$ $\operatorname{span}\left\{C_{i}-\boldsymbol{x}_{i}\right\}$, then $\operatorname{dim}\left(L_{i}\right)=\operatorname{dim}\left(\operatorname{conv} C_{i}\right)$. Clearly conv $C \subset \boldsymbol{x}+\left(L_{1}+\right.$ $\left.\cdots+L_{n}\right)$, so $\operatorname{dim}(\operatorname{conv} C) \leq \operatorname{dim}\left(L_{1}+\cdots+L_{n}\right)$. By condition $(\mathbf{d} 1)$ in the 
definition of mixed subdivision,

$$
\operatorname{dim}(\operatorname{conv} C)=n=\sum_{i=1}^{n} \operatorname{dim}\left(\operatorname{conv} C_{i}\right)=\sum_{i=1}^{n} \operatorname{dim}\left(L_{i}\right)
$$

Therefore the subspaces $L_{1}, \ldots, L_{n}$ must be linearly independent. But $\boldsymbol{x}_{i}^{\prime}-$ $\boldsymbol{x}_{i} \in L_{i}$ for each $i=1, \ldots, n$ and $\lambda_{1}\left(\boldsymbol{x}_{1}^{\prime}-\boldsymbol{x}_{1}\right)+\cdots+\lambda_{n}\left(\boldsymbol{x}_{n}^{\prime}-\boldsymbol{x}_{n}\right)=\mathbf{0}$, so each $\lambda_{i}\left(\boldsymbol{x}_{i}^{\prime}-\boldsymbol{x}_{i}\right)$ must be zero and hence $\boldsymbol{x}_{i}^{\prime}=\boldsymbol{x}_{i}$ for each $i=1, \ldots, n$.

Taking $\boldsymbol{\lambda}=(1, \ldots, 1)$, this lemma implies that for any cell $C=\left(C_{1}, \ldots\right.$, $\left.C_{n}\right) \in \mathcal{D}$, each point $\boldsymbol{x} \in \operatorname{conv} C$ has a unique representation as a sum $\boldsymbol{x}=$ $\boldsymbol{x}_{1}+\cdots+\boldsymbol{x}_{n}$ with $\boldsymbol{x}_{i} \in \operatorname{conv} C_{i}$ for $i=1, \ldots, n$. So, the scaling map $\phi_{\boldsymbol{\lambda}}^{\mathcal{D}}$ : $\operatorname{conv} C \rightarrow \operatorname{conv}(\boldsymbol{\lambda} \circ C)$ given by

$$
\phi_{\boldsymbol{\lambda}}^{\mathcal{D}}(\boldsymbol{x}):=\lambda_{1} \boldsymbol{x}_{1}+\cdots+\lambda_{n} \boldsymbol{x}_{n}, \quad \text { for } \boldsymbol{x}=\boldsymbol{x}_{1}+\cdots+\boldsymbol{x}_{n}
$$

is well defined as a nonsingular affine map.

Lemma 8. Let $A=\left(A_{1}, \ldots, A_{n}\right), B=\left(B_{1}, \ldots, B_{n}\right) \in \mathcal{D}$. If $\boldsymbol{a}_{1}+\cdots+\boldsymbol{a}_{n} \in$ $\operatorname{conv} A \cap \operatorname{conv} B$ with $\boldsymbol{a}_{1} \in \operatorname{conv} A_{1}, \ldots, \boldsymbol{a}_{n} \in \operatorname{conv} A_{n}$ then $\boldsymbol{a}_{1} \in \operatorname{conv} B_{1}, \ldots$, $\boldsymbol{a}_{n} \in \operatorname{conv} B_{n}$.

Proof. Since $\boldsymbol{a}_{1}+\cdots+\boldsymbol{a}_{n} \in \operatorname{conv} A \cap \operatorname{conv} B=\sum_{i=1}^{n}\left(\operatorname{conv} A_{i} \cap \operatorname{conv} B_{i}\right)$ by condition (d2) in the definition of mixed subdivision, and conv $A_{i} \cap \operatorname{conv} B_{i} \subseteq$ conv $A_{i}$ for each $i=1, \ldots, n$, hence $\boldsymbol{a}_{i} \in \operatorname{conv} A_{i} \cap$ conv $B_{i}$ because the representation of $\boldsymbol{a}_{1}+\cdots+\boldsymbol{a}_{n}$ in conv $A$ must be unique by Lemma 7 . Therefore, for each $i=1, \ldots, n, \boldsymbol{a}_{i} \in \operatorname{conv} B_{i}$.

By this lemma, for any point on the common face of two cells, its representations in both cells must be identical, which, along with Lemma 7 , implies that every point $\boldsymbol{x} \in \widetilde{\mathcal{Q}}=\mathcal{Q}_{1}+\cdots+\mathcal{Q}_{n}$ has a unique representation of the form $\boldsymbol{x}_{1}+\cdots+\boldsymbol{x}_{n}$ with $\boldsymbol{x}_{i} \in \mathcal{Q}_{i}$. Therefore the scaling map (1) can be extended to a well defined continuous map $\phi_{\boldsymbol{\lambda}}^{\mathcal{D}}: \widetilde{\mathcal{Q}} \rightarrow \boldsymbol{\lambda} \circ \widetilde{\mathcal{Q}}=\lambda_{1} \mathcal{Q}_{1}+$ $\cdots+\lambda_{n} \mathcal{Q}_{n}$ by

$$
\phi_{\boldsymbol{\lambda}}^{\mathcal{D}}(\boldsymbol{x})=\lambda_{1} \boldsymbol{x}_{1}+\cdots+\lambda_{n} \boldsymbol{x}_{n}
$$

where $\boldsymbol{x}=\boldsymbol{x}_{1}+\cdots+\boldsymbol{x}_{n}$ with $\boldsymbol{x}_{i} \in \mathcal{Q}_{i}$ is the unique representation of $\boldsymbol{x}$ given by the mixed subdivision $\mathcal{D}$. A direct consequence is that under scaling neighboring cells remain neighbors:

Lemma 9. For $A, B \in \mathcal{D}$, if $(\operatorname{conv} A) \cap(\operatorname{conv} B) \neq \varnothing$, then $\operatorname{conv}(\boldsymbol{\lambda} \circ A) \cap$ $\operatorname{conv}(\boldsymbol{\lambda} \circ B) \neq \varnothing$. 
Proof. For $A=\left(A_{1}, \ldots, A_{n}\right)$ and $B=\left(B_{1}, \ldots, B_{n}\right)$, let $\boldsymbol{a} \in \operatorname{conv} A \cap \operatorname{conv} B$, then $\boldsymbol{a}=\boldsymbol{a}_{1}+\cdots+\boldsymbol{a}_{n}$ for some $\boldsymbol{a}_{i} \in \operatorname{conv} A_{i}$ for $i=1, \ldots, n$. But by the previous lemma, each $\boldsymbol{a}_{i} \in \operatorname{conv} B_{i}$. Thus for $i=1, \ldots, n, \lambda_{i} \boldsymbol{a}_{i}$ is in both conv $\lambda_{i} A_{i}$ and conv $\lambda_{i} B_{i}$. Therefore $\phi_{\boldsymbol{\lambda}}^{\mathcal{D}}(\boldsymbol{a}) \in \operatorname{conv}(\boldsymbol{\lambda} \circ A) \cap \operatorname{conv}(\boldsymbol{\lambda} \circ B)$, and it cannot be empty.

Defined in terms of the mixed subdivision $\mathcal{D}$, the scaling map $\phi_{\boldsymbol{\lambda}}^{\mathcal{D}}$ is well defined and continuous on all of $\widetilde{\mathcal{Q}}=\mathcal{Q}_{1}+\cdots+\mathcal{Q}_{n}$.

Lemma 10. Let $A=\left(A_{1}, \ldots, A_{n}\right), B=\left(B_{1}, \ldots, B_{n}\right) \in \mathcal{D}$ with conv $A \cap$ $\operatorname{conv} B \neq \varnothing$. If $\boldsymbol{a} \in \operatorname{conv} A, \boldsymbol{b} \in \operatorname{conv} B$ and $\boldsymbol{a} \neq \boldsymbol{b}$, then $\phi_{\boldsymbol{\lambda}}^{\mathcal{D}}(\boldsymbol{a}) \neq \phi_{\boldsymbol{\lambda}}^{\mathcal{D}}(\boldsymbol{b})$.

Proof. If both $\boldsymbol{a}$ and $\boldsymbol{b}$ are in conv $A \cap \operatorname{conv} B$, then the agreement of $\phi_{\boldsymbol{\lambda}}^{\mathcal{D}}(\boldsymbol{a})$ and $\phi_{\boldsymbol{\lambda}}^{\mathcal{D}}(\boldsymbol{b})$, both in $\operatorname{conv}(\boldsymbol{\lambda} \circ A)$, would contradict to the unique representation of $\phi_{\boldsymbol{\lambda}}^{\mathcal{D}}(\boldsymbol{a})$ provided by Lemma 7 .

On the other hand, if $\boldsymbol{a} \notin \operatorname{conv} A \cap \operatorname{conv} B$, let $\boldsymbol{r}=\boldsymbol{r}_{1}+\cdots+\boldsymbol{r}_{n}$ be any point in $(\operatorname{conv} A) \cap(\operatorname{conv} B)$. Then, since $(\operatorname{conv} A) \cap(\operatorname{conv} B)$ is a face of both conv $A$ and conv $B$, passing through $\boldsymbol{r}$ there exists a hyperplane $H$ defined by $\langle\boldsymbol{\alpha}, \bullet\rangle=h$ for some $\boldsymbol{\alpha} \in \mathbb{R}^{n}$ and $h \in \mathbb{R}$ such that $\operatorname{int}(\operatorname{conv} A)$ and $\operatorname{int}(\operatorname{conv} B)$ are in $H^{-}:=\left\{\boldsymbol{x} \in \mathbb{R}^{n} \mid\langle\boldsymbol{\alpha}, \boldsymbol{x}\rangle\langle h\}\right.$ and $H^{+}:=\left\{\boldsymbol{x} \in \mathbb{R}^{n}|\langle\boldsymbol{\alpha}, \boldsymbol{x}\rangle\rangle\right.$ $h\}$ respectively. Also, $\boldsymbol{r} \in(\operatorname{conv} A)_{\boldsymbol{\alpha}}$ and $\boldsymbol{r} \in(\operatorname{conv} B)_{-\boldsymbol{\alpha}}$, hence by linearity, $\boldsymbol{r}_{i} \in\left(\operatorname{conv} A_{i}\right)_{\boldsymbol{\alpha}}$ and $\boldsymbol{r}_{i} \in\left(\operatorname{conv} B_{i}\right)_{-\boldsymbol{\alpha}}$ for each $i=1, \ldots, n$. That is, with $\boldsymbol{a}=\boldsymbol{a}_{1}+\cdots+\boldsymbol{a}_{n}$ and $\boldsymbol{b}=\boldsymbol{b}_{1}+\cdots+\boldsymbol{b}_{n}$,

$$
\left\langle\boldsymbol{\alpha}, \boldsymbol{a}_{i}\right\rangle>\left\langle\boldsymbol{\alpha}, \boldsymbol{r}_{i}\right\rangle \geqslant\left\langle\boldsymbol{\alpha}, \boldsymbol{b}_{i}\right\rangle
$$

for each $i=1, \ldots, n$. Therefore

$$
\langle\boldsymbol{\alpha}, \boldsymbol{\lambda} \circ \boldsymbol{a}\rangle=\sum_{i=1}^{n} \lambda_{i}\left\langle\boldsymbol{\alpha}, \boldsymbol{a}_{i}\right\rangle>\sum_{i=1}^{n} \lambda_{i}\left\langle\boldsymbol{\alpha}, \boldsymbol{r}_{i}\right\rangle \geqslant \sum_{i=1}^{n} \lambda_{i}\left\langle\boldsymbol{\alpha}, \boldsymbol{b}_{i}\right\rangle=\langle\boldsymbol{\alpha}, \boldsymbol{\lambda} \circ \boldsymbol{b}\rangle .
$$

So, $\boldsymbol{\lambda} \circ \boldsymbol{a} \neq \boldsymbol{\lambda} \circ \boldsymbol{b}$, that is, $\phi_{\boldsymbol{\lambda}}^{\mathcal{D}}(\boldsymbol{a}) \neq \phi_{\boldsymbol{\lambda}}^{\mathcal{D}}(\boldsymbol{b})$.

Consequently, fix a cell $A \in \mathcal{D}$, for any neighboring cell $B \in \mathcal{D}$, the continuous map $\phi_{\boldsymbol{\lambda}}^{\mathcal{D}}$ is also one-to-one on $(\operatorname{conv} A) \cup(\operatorname{conv} B)$.

With these lemmas ready, we now prove Proposition 4.

Proof of Proposition 4. Let $\mathcal{D}$ be a (fine) mixed subdivision of the $n$-tuple $\mathcal{S}=\left(\mathcal{S}_{1}, \ldots, \mathcal{S}_{n}\right)$, and $\boldsymbol{\lambda}=\left(\lambda_{1}, \ldots, \lambda_{n}\right) \in\left(\mathbb{R}^{+}\right)^{n}$. For the proof of $\boldsymbol{\lambda} \circ \mathcal{D}:=$ $\left\{\boldsymbol{\lambda} \circ C=\left(\lambda_{1} C_{1}, \ldots, \lambda_{n} C_{n}\right): C=\left(C_{1}, \ldots, C_{n}\right) \in \mathcal{D}\right\}$ being a (fine) mixed subdivision of $\boldsymbol{\lambda} \circ \mathcal{S}=\left(\lambda_{1} \mathcal{S}_{1}, \ldots, \lambda_{n} \mathcal{S}_{n}\right)$, conditions (a), (d1), and (e) in 
Definitions 1, 2, and 3 are clearly valid since for a cell $C=\left(C_{1}, \ldots, C_{n}\right) \in \mathcal{D}$, the scaling conv $C_{i} \mapsto \operatorname{conv}\left(\lambda_{i} C_{i}\right)$, for each $i=1, \ldots, n$ is a nonsingular affine transformation which preserves dimensions of affine spaces. Below we verify that conditions (b), (c), and (d2) hold.

(b) For condition (b), let $A=\left(A_{1}, \ldots, A_{n}\right)$ and $B=\left(B_{1}, \ldots, B_{n}\right)$ be two cells in $\mathcal{D}$. We first show that $\operatorname{conv}(\boldsymbol{\lambda} \circ A) \cap \operatorname{conv}(\boldsymbol{\lambda} \circ B) \neq \varnothing$ yields $(\operatorname{conv} A) \cap(\operatorname{conv} B) \neq \varnothing$. Suppose that $\operatorname{conv} A \cap \operatorname{conv} B=\varnothing$. For $\boldsymbol{x} \in$ conv $\widetilde{\mathcal{Q}}$ and $s \in[0,1]$, define

$$
h(\boldsymbol{x}, s):=\phi_{[(1-s) \boldsymbol{\lambda}+s \mathbf{1}]}^{\mathcal{D}}(\boldsymbol{x})
$$

where $\mathbf{1}=(1, \ldots, 1)$. By the continuity we established for the map $\phi_{\boldsymbol{\lambda}}^{\mathcal{D}}: \widetilde{\mathcal{Q}} \rightarrow \boldsymbol{\lambda} \circ \widetilde{\mathcal{Q}}$ before, $h(\boldsymbol{x}, s)$ is continuous for each $s \in[0,1]$. Furthermore, since $H(\boldsymbol{x}, 1)=\boldsymbol{x}$ and $H(\boldsymbol{x}, 0)=\phi_{\boldsymbol{\lambda}}^{\mathcal{D}}(\boldsymbol{x})$, so

$h(\operatorname{conv} A, 1) \cap H(\operatorname{conv} B, 1)=(\operatorname{conv} A) \cap(\operatorname{conv} B)=\varnothing \quad$ and $h(\operatorname{conv} A, 0) \cap H(\operatorname{conv} B, 0)=\operatorname{conv}(\boldsymbol{\lambda} \circ A) \cap \operatorname{conv}(\boldsymbol{\lambda} \circ B) \neq \varnothing$.

Thus, there exits $s_{0} \in(0,1)$, for which

$$
\begin{aligned}
h\left(\operatorname{conv} A, s_{0}\right) \cap H\left(\operatorname{conv} B, s_{0}\right) \neq \varnothing & \text { and } \\
h(\operatorname{conv} A, s) \cap H(\operatorname{conv} B, s)=\varnothing & \text { for } 0<s_{0}<s \leq 1 .
\end{aligned}
$$

Let $N:=\{C \in \mathcal{D}: \operatorname{conv} C \cap \operatorname{conv} A \neq \varnothing\}$, and

$$
G=\left(\bigcup_{C \in N} \operatorname{conv} C\right), \quad T=\partial G \backslash \partial(\operatorname{conv} \mathcal{S}) .
$$

Geometrically, $T$ may be considered a "shell" that separates conv $A$ and conv $B$. By Lemma 9, neighboring cells remain neighbors after the mixed scaling, so $h(T, s)$ remains connected for all $s \in[0,1]$. Therefore as $s$ decreases from 1 , since $h(\operatorname{conv} A, s)$ and $h(\operatorname{conv} B, s)$ will meet at $s_{0}, h(\operatorname{conv} A, s)$ and $h(T, s)$ must intersect at some $s_{1} \in\left[s_{0}, 1\right]$. But $T$ consists of boundaries of neighbors of $A$, so there is a neighbor cell $C$ of $A$ in $\mathcal{D}$ and a point $\boldsymbol{x} \in \operatorname{conv} C \backslash \operatorname{conv} A$ such that $\phi_{\left[\left(1-s_{1}\right) \boldsymbol{\lambda}+s \mathbf{1}\right]}^{\mathcal{D}}(\boldsymbol{x}) \in$ $\phi_{\left[\left(1-s_{1}\right) \boldsymbol{\lambda}+s \mathbf{1}\right]}^{\mathcal{D}}(\operatorname{conv} A)$. This violates Lemma 10 . Therefore $(\operatorname{conv} A) \cap$ $(\operatorname{conv} B) \neq \varnothing$. 
Now, since $(\operatorname{conv} A) \cap(\operatorname{conv} B) \neq \varnothing$ is a common face of both conv $A$ and $\operatorname{conv} B$, by the hyperplane separation theorem, there exists a nonzero vector $\boldsymbol{\alpha}$ such that

$$
(\operatorname{conv} A) \cap(\operatorname{conv} B)=(\operatorname{conv} A)_{\boldsymbol{\alpha}}=(\operatorname{conv} B)_{-\boldsymbol{\alpha}}
$$

We claim that a hyperplane defined by $\boldsymbol{\alpha}$ separates $\operatorname{conv}(\boldsymbol{\lambda} \circ A)$ and $\operatorname{conv}(\boldsymbol{\lambda} \circ B)$. To prove this, let $\boldsymbol{a}^{\prime} \in(\operatorname{conv} \boldsymbol{\lambda} \circ A)_{\boldsymbol{\alpha}}$, then $\boldsymbol{a}^{\prime}=\lambda_{1} \boldsymbol{a}_{1}+$ $\cdots+\lambda_{n} \boldsymbol{a}_{n}$ for some $\boldsymbol{a}_{1} \in \operatorname{conv} A_{1}, \ldots, \boldsymbol{a}_{n} \in \operatorname{conv} A_{n}$. By linearity, $\boldsymbol{a}_{i} \in$ $\left(\operatorname{conv} A_{i}\right)_{\boldsymbol{\alpha}}$ for each $i=1, \ldots, n$, and hence $\boldsymbol{a}_{1}+\cdots+\boldsymbol{a}_{n} \in(\operatorname{conv} A)_{\boldsymbol{\alpha}}$ $=\operatorname{conv} A \cap \operatorname{conv} B$. By Lemma $8, \boldsymbol{a}_{i} \in \operatorname{conv} B_{i}$ for each $i$, and therefore $\boldsymbol{a}^{\prime}=\lambda_{1} \boldsymbol{a}_{1}+\cdots+\lambda_{n} \boldsymbol{a}_{n} \in \operatorname{conv}(\boldsymbol{\lambda} \circ B)$. This shows $(\operatorname{conv} \boldsymbol{\lambda} \circ A)_{\boldsymbol{\alpha}} \subset$ conv $\boldsymbol{\lambda} \circ B$. Secondly, if $\boldsymbol{a}^{\prime} \in \operatorname{conv}(\boldsymbol{\lambda} \circ A) \backslash(\operatorname{conv} \boldsymbol{\lambda} \circ A)_{\boldsymbol{\alpha}}$, by Lemma 7 there exists a unique list $\boldsymbol{a}_{1} \in \operatorname{conv} A_{1}, \ldots, \boldsymbol{a}_{n} \in \operatorname{conv} A_{n}$ such that $\boldsymbol{a}^{\prime}=\lambda_{1} \boldsymbol{a}_{1}+\cdots+\lambda_{n} \boldsymbol{a}_{n}$. Then $\boldsymbol{a}_{1}+\cdots+\boldsymbol{a}_{n} \in \operatorname{conv} A \backslash \operatorname{conv} B$. By Lemma 10, $\boldsymbol{a}^{\prime} \notin \operatorname{conv}(\boldsymbol{\lambda} \circ B)$. Combining those two parts shows that the subset of $\operatorname{conv}(\boldsymbol{\lambda} \circ A)$ inside $\operatorname{conv}(\boldsymbol{\lambda} \circ B)$ is exactly $(\operatorname{conv}(\boldsymbol{\lambda} \circ A))_{\boldsymbol{\alpha}}$.

By a symmetric argument, the only subset of $\operatorname{conv}(\boldsymbol{\lambda} \circ B)$ inside $\operatorname{conv}(\boldsymbol{\lambda} \circ A)$ is exactly $(\operatorname{conv}(\boldsymbol{\lambda} \circ B))_{-\boldsymbol{\alpha}}$. Therefore $\operatorname{conv}(\boldsymbol{\lambda} \circ A) \cap$ conv $(\boldsymbol{\lambda} \circ B)=(\operatorname{conv}(\boldsymbol{\lambda} \circ A))_{\boldsymbol{\alpha}}=(\operatorname{conv}(\boldsymbol{\lambda} \circ B))_{-\boldsymbol{\alpha}}$ is a common face of both.

(c) We need to show $M:=\bigcup_{C \in \mathcal{D}} \operatorname{conv}(\boldsymbol{\lambda} \circ C)=\boldsymbol{\lambda} \circ \widetilde{\mathcal{Q}}:=\lambda_{1} \mathcal{Q}_{1}+\cdots+$ $\lambda_{n} \mathcal{Q}_{n}$ where $\mathcal{Q}_{i}=\operatorname{conv} \mathcal{S}_{i}$ for $i=1, \ldots, n$. One direction of the containment is obvious: since for each $C \in \mathcal{D}, \operatorname{conv}(\boldsymbol{\lambda} \circ C)=\boldsymbol{\lambda} \circ \operatorname{conv} C \subseteq$ $\boldsymbol{\lambda} \circ \widetilde{\mathcal{Q}}$, the union is still in $\boldsymbol{\lambda} \circ \widetilde{\mathcal{Q}}$. For the other direction, suppose $M \subsetneq \boldsymbol{\lambda} \circ \widetilde{\mathcal{Q}}$, then $M$ has boundary points outside $\partial(\boldsymbol{\lambda} \circ \widetilde{\mathcal{Q}})$. Let $\boldsymbol{q} \in$ $\partial M \backslash \partial(\boldsymbol{\lambda} \circ \widetilde{\mathcal{Q}})$. Then, $\boldsymbol{q}$ is in

$$
\partial \bigcup_{C \in \mathcal{D}} \operatorname{conv}(\boldsymbol{\lambda} \circ C) \backslash \partial(\boldsymbol{\lambda} \circ \widetilde{\mathcal{Q}}) \subseteq \bigcup_{C \in \mathcal{D}} \partial(\operatorname{conv}(\boldsymbol{\lambda} \circ C)) \backslash \partial(\boldsymbol{\lambda} \circ \widetilde{\mathcal{Q}})
$$

and there exists a cell $C=\left(C_{1}, \ldots, C_{n}\right) \in \mathcal{D}$ such that $\boldsymbol{q} \in \partial(\operatorname{conv}(\boldsymbol{\lambda} \circ$ $C)$ ). Without loss of generality, assume $\boldsymbol{q}$ lies on a facet of $\operatorname{conv}(\boldsymbol{\lambda} \circ C)$ but not on any other of its faces. By Lemma 7, there is a unique list $\boldsymbol{q}_{1} \in \operatorname{conv} C_{1}, \ldots, \boldsymbol{q}_{n} \in \operatorname{conv} C_{n}$ such that $\boldsymbol{q}=\lambda_{1} \boldsymbol{q}_{1}+\cdots+\lambda_{n} \boldsymbol{q}_{n}$. Then $\boldsymbol{q}_{1}+\cdots+\boldsymbol{q}_{n}$ must be on a facet of conv $C$ but not on any other of its faces.

If $\boldsymbol{q}_{1}+\cdots+\boldsymbol{q}_{n} \in \partial \widetilde{\mathcal{Q}}$ then $\boldsymbol{q}_{1}+\cdots+\boldsymbol{q}_{n} \in \mathcal{Q}_{\boldsymbol{\alpha}}=\left(\mathcal{Q}_{1}\right)_{\boldsymbol{\alpha}}+\cdots+$ $\left(\mathcal{Q}_{n}\right)_{\boldsymbol{\alpha}}$ for some nonzero $\boldsymbol{\alpha} \in \mathbb{R}^{n}$ and hence 


$$
\begin{aligned}
\boldsymbol{q} & =\lambda_{1} \boldsymbol{q}_{1}+\cdots+\lambda_{n} \boldsymbol{q}_{n} \\
& \in \lambda_{1}\left(\mathcal{Q}_{1}\right)_{\boldsymbol{\alpha}}+\cdots+\lambda_{n}\left(\mathcal{Q}_{n}\right)_{\boldsymbol{\alpha}} \\
& =(\boldsymbol{\lambda} \circ \widetilde{\mathcal{Q}})_{\boldsymbol{\alpha}} \\
& \subset \partial(\boldsymbol{\lambda} \circ \widetilde{\mathcal{Q}})
\end{aligned}
$$

a contradiction. So, $\boldsymbol{q}_{1}+\cdots+\boldsymbol{q}_{n} \notin \partial \widetilde{\mathcal{Q}}$. Therefore $\boldsymbol{q}_{1}+\cdots+\boldsymbol{q}_{n}$ must be on a common facet of $\operatorname{conv} C$ and $\operatorname{conv} C^{\prime}$ for some other cell $C^{\prime}=$ $\left(C_{1}^{\prime}, \ldots, C_{n}^{\prime}\right)$. By Lemma $8, \boldsymbol{q}_{1} \in \mathrm{conv} C_{1}^{\prime}, \ldots, \boldsymbol{q}_{n} \in \operatorname{conv} C_{n}^{\prime}$, and therefore $\boldsymbol{q}=\lambda_{1} \boldsymbol{q}_{1}+\cdots+\lambda_{n} \boldsymbol{q}_{n}$ is in both $\operatorname{conv}(\boldsymbol{\lambda} \circ C)$ and $\operatorname{conv}\left(\boldsymbol{\lambda} \circ C^{\prime}\right)$. Indeed, $\boldsymbol{q}$ is in the interior of $\operatorname{conv}(\boldsymbol{\lambda} \circ C) \cup \operatorname{conv}\left(\boldsymbol{\lambda} \circ C^{\prime}\right)$, contradicting to the assumption that $\boldsymbol{q} \in \partial M$. Therefore we can only conclude that $M=\boldsymbol{\lambda} \circ \widetilde{\mathcal{Q}}$.

(d2) Let $A=\left(A_{1}, \ldots, A_{n}\right)$ and $B=\left(B_{1}, \ldots, B_{n}\right)$ be cells in $\mathcal{D}$. By condition (b), $\operatorname{conv}(\boldsymbol{\lambda} \circ A) \cap \operatorname{conv}(\boldsymbol{\lambda} \circ B)$, if nonempty, is a common face of both of them, i.e., the intersection is of the form $(\operatorname{conv}(\boldsymbol{\lambda} \circ A))_{\boldsymbol{\alpha}}$ for $\boldsymbol{\alpha} \in \mathbb{R}^{n}$. So

$$
\begin{aligned}
\operatorname{conv}(\boldsymbol{\lambda} \circ A) \cap \operatorname{conv}(\boldsymbol{\lambda} \circ B) & =(\operatorname{conv}(\boldsymbol{\lambda} \circ A))_{\boldsymbol{\alpha}} \\
& =\left(\sum_{i=1}^{n} \operatorname{conv} \lambda_{i} A_{i}\right)_{\boldsymbol{\alpha}} \\
& =\sum_{i=1}^{n} \lambda_{i} \operatorname{conv}\left(A_{i}\right)_{\boldsymbol{\alpha}} \\
& =\sum_{i=1}^{n} \lambda_{i}\left(\operatorname{conv} A_{i} \cap \operatorname{conv} B_{i}\right) .
\end{aligned}
$$

\section{Computing mixed volume via fine mixed subdivisions}

Once a fine mixed subdivision $\mathcal{D}$ of $\mathcal{S}=\left(\mathcal{S}_{1}, \ldots, \mathcal{S}_{n}\right)$ is available, the mixed volume $\mathcal{M}\left(\mathcal{Q}_{1}, \ldots, \mathcal{Q}_{n}\right)$ with $\mathcal{Q}_{i}=\operatorname{conv} \mathcal{S}_{i}$ can now be computed. This section describes the concrete procedure and provides its theoretical justification.

We begin with the volume change of a cell $C \in \mathcal{D}$ under mixed scaling by $\boldsymbol{\lambda}=\left(\lambda_{1}, \ldots, \lambda_{n}\right)$. 
Proposition 11 (Cell volume under mixed scaling). If $\mathcal{D}$ is a fine mixed subdivision, and a cell $C=\left(C_{1}, \ldots, C_{n}\right) \in \mathcal{D}$ is of type $\left(k_{1}, \ldots, k_{n}\right)$, then for $\boldsymbol{\lambda}=\left(\lambda_{1}, \ldots, \lambda_{n}\right) \in\left(\mathbb{R}^{+}\right)^{n}$

$$
\operatorname{Vol}_{n}(\boldsymbol{\lambda} \circ C)=\lambda_{1}^{k_{1}} \ldots \lambda_{n}^{k_{n}} \operatorname{Vol}_{n}(C) .
$$

Proof. Recall that for a mixed cell $C=\left(C_{1}, \ldots, C_{n}\right)$ in the fine mixed subdivision $\mathcal{D}$ with type $(C)=\left(k_{1}, \ldots, k_{n}\right)$, where $k_{i}=\operatorname{dim}\left(\operatorname{conv} C_{i}\right)$, we have, by definition, $k_{1}+\cdots+k_{n}=n$, and each conv $C_{i}$ is a simplex of dimension $k_{i}=\# C_{i}-1$. Write $C_{i}=\left\{\boldsymbol{c}_{0}^{i}, \ldots, \boldsymbol{c}_{k_{i}}^{i}\right\}$ for each $i=1, \ldots, n$. We shall construct an explicit parametrization of points in $\operatorname{conv}(\boldsymbol{\lambda} \circ C)$.

Any point $\boldsymbol{x} \in \operatorname{conv}(\boldsymbol{\lambda} \circ C)$ can be written, by Lemma 7 , as $\lambda_{1} \boldsymbol{x}_{1}+\cdots+$ $\lambda_{n} \boldsymbol{x}_{n}$ for a unique list of $\boldsymbol{x}_{i} \in \operatorname{conv} C_{i}$ for $i=1, \ldots, n$. For a fixed $i$, the point $\boldsymbol{x}_{i}$ can, in turn, be written as a convex combination of $\left\{\boldsymbol{c}_{0}^{i}, \ldots, \boldsymbol{c}_{k_{i}}^{i}\right\}$, i.e., there exist $t_{i, 0}, \ldots, t_{i, k_{i}} \geq 0$ and $\sum_{j=0}^{k_{i}} t_{i, j}=1$ such that $\boldsymbol{x}_{i}=\sum_{j=0}^{k_{i}} t_{i, j} \boldsymbol{c}_{j}^{i}$. Therefore

$$
\boldsymbol{x}=\sum_{i=1}^{n}\left(\lambda_{i} \sum_{j=0}^{k_{i}} t_{i, j} \boldsymbol{c}_{j}^{i}\right)
$$

Rearranging terms yields

$$
\boldsymbol{x}=\sum_{i=1}^{n} \lambda_{i}\left[\sum_{j=0}^{k_{i}} t_{i, j} \boldsymbol{c}_{0}^{i}+\sum_{j=1}^{k_{i}} t_{i, j}\left(\boldsymbol{c}_{j}^{i}-\boldsymbol{c}_{0}^{i}\right)\right]=\sum_{i=1}^{n} \lambda_{i}\left[\boldsymbol{c}_{0}^{i}+\sum_{j=1}^{k_{i}} t_{i, j}\left(\boldsymbol{c}_{j}^{i}-\boldsymbol{c}_{0}^{i}\right)\right] .
$$

For each $i=1, \ldots, n$, define the $n \times k_{i}$ (empty when $\left.k_{i}=0\right)$ matrix

$$
V\left(C_{i}\right):=\left[\begin{array}{lll}
\boldsymbol{c}_{1}^{i}-\boldsymbol{c}_{0}^{i} & \cdots & \boldsymbol{c}_{k_{i}}^{i}-\boldsymbol{c}_{0}^{i}
\end{array}\right]
$$

With these, define the block matrices

$$
\begin{aligned}
V(C) & =\left[\begin{array}{lll}
V\left(C_{1}\right) & \cdots & V\left(C_{n}\right)
\end{array}\right] \\
V_{\boldsymbol{\lambda}}(C) & =\left[\begin{array}{lll}
\lambda_{1} \cdot V\left(C_{1}\right) & \cdots & \lambda_{n} \cdot V\left(C_{n}\right)
\end{array}\right]
\end{aligned}
$$

which are both of size $n \times\left(k_{1}+\cdots+k_{n}\right)=n \times n$ (if $k_{i}=0$ then the block $V\left(C_{i}\right)$ does not appear). We may now rewrite (4) as

(8) $\boldsymbol{x}=\left(\sum_{i=1}^{n} \boldsymbol{c}_{0}^{i}\right)+V(C) \cdot\left[\begin{array}{c}\boldsymbol{t}_{1} \\ \vdots \\ \boldsymbol{t}_{n}\end{array}\right]$ where $\boldsymbol{t}_{i}=\left[\begin{array}{c}t_{i, 1} \\ \vdots \\ t_{i, k_{i}}\end{array}\right]$ for $i=1, \ldots, n$.

Again, when $k_{i}=0, \boldsymbol{t}_{i}$ will be absent in $\left(\boldsymbol{t}_{1}, \ldots, \boldsymbol{t}_{n}\right)^{T}$. Recall that for each $i, t_{i, 0} \geq 0, \ldots, t_{i, k_{i}} \geq 0$ and $\sum_{j=0}^{k_{i}} t_{i, j}=1$, hence $0 \leq \sum_{j=1}^{k_{i}} t_{i, j} \leq 1$. So, each 
$\boldsymbol{t}_{i}=\left(t_{i, 1}, \ldots, t_{i, k_{i}}\right)^{T}$ is a point in the standard $k_{i}$-simplex $\Delta_{k_{i}}$ (which would be a single point if $k_{i}=0$ ). Thus, the affine map $T: \Delta_{k_{1}} \times \cdots \times \Delta_{k_{n}} \rightarrow \mathbb{R}^{n}$ given by

$$
T\left(\boldsymbol{t}_{1}, \ldots, \boldsymbol{t}_{n}\right)=\left(\sum_{i=1}^{n} \lambda_{i} \boldsymbol{c}_{0}^{i}\right)+V(C) \cdot\left[\begin{array}{c}
\boldsymbol{t}_{1} \\
\vdots \\
\boldsymbol{t}_{n}
\end{array}\right]
$$

makes every $\boldsymbol{x} \in \operatorname{conv}(\boldsymbol{\lambda} \circ C)$ a point in the image of $T$. It is clear that the converse is also true: For every point $\left(\boldsymbol{t}_{1}, \ldots, \boldsymbol{t}_{n}\right) \in \Delta_{k_{1}} \times \cdots \times \Delta_{k_{n}}$, $T\left(\boldsymbol{t}_{1}, \ldots, \boldsymbol{t}_{n}\right)$ lies in $\operatorname{conv}(\boldsymbol{\lambda} \circ C)$. Furthermore, by the uniqueness of representation given by Lemma 7, $T$ is also one-to-one. Therefore, $\operatorname{conv}(\boldsymbol{\lambda} \circ C)$ is precisely the image of $\Delta_{k_{1}} \times \cdots \times \Delta_{k_{n}}$ under the affine transformation $T$, and hence

$$
\operatorname{Vol}_{n}(\boldsymbol{\lambda} \circ C)=\operatorname{Vol}_{n}(\operatorname{conv}(\boldsymbol{\lambda} \circ C))=\left|\operatorname{det} V_{\boldsymbol{\lambda}}(C)\right| \cdot \operatorname{Vol}_{n}\left(\Delta_{k_{1}} \times \cdots \times \Delta_{k_{n}}\right)
$$

where $V_{\boldsymbol{\lambda}}(C)$, defined in $(7)$, is the Jacobian matrix of $T$. Since $\operatorname{Vol}_{n}\left(\Delta_{k_{i}}\right)=$ $\frac{1}{k_{i} !}$, so $\operatorname{Vol}_{n}\left(\Delta_{k_{1}} \times \cdots \times \Delta_{k_{n}}\right)=\frac{1}{k_{1} ! \cdots k_{n} !}$. By (7),

$$
\begin{aligned}
\operatorname{det} V_{\boldsymbol{\lambda}}(C) & =\operatorname{det}\left[\begin{array}{lll}
\lambda_{1} \cdot V_{1}(C) & \cdots & \lambda_{n} \cdot V_{n}(C)
\end{array}\right] \\
& =\lambda_{1}^{k_{1}} \cdots \lambda_{n}^{k_{n}} \operatorname{det}\left[\begin{array}{lll}
V_{1}(C) & \cdots & V_{n}(C)
\end{array}\right] \\
& =\lambda_{1}^{k_{1}} \cdots \lambda_{n}^{k_{n}} \operatorname{det} V(C)
\end{aligned}
$$

since each $V_{i}(C)$ is a block of size $n \times k_{i}$. It follows that

$$
\operatorname{Vol}_{n}(\boldsymbol{\lambda} \circ C)=\frac{\lambda_{1}^{k_{1}} \cdots \lambda_{n}^{k_{n}}}{k_{1} ! \cdots k_{n} !}|\operatorname{det} V(C)| .
$$

Taking $\lambda=(1, \ldots, 1)$ yields the special case

$$
\operatorname{Vol}_{n}(C)=\frac{1}{k_{1} ! \cdots k_{n} !} \cdot|\operatorname{det} V(C)| \text {. }
$$

The assertion of the proposition thus follows by comparing the two equations above. 
Proposition 12. If $\mathcal{D}$ is a fine mixed subdivision of $\mathcal{S}=\left(\mathcal{S}_{1}, \ldots, \mathcal{S}_{n}\right)$, and $\boldsymbol{\lambda}=\left(\lambda_{1}, \ldots, \lambda_{n}\right) \in\left(\mathbb{R}^{+}\right)^{n}$, then, with $\mathcal{Q}_{i}=\operatorname{conv} \mathcal{S}_{i}$,

$$
\operatorname{Vol}_{n}\left(\lambda_{1} \mathcal{Q}_{1}+\cdots+\lambda_{n} \mathcal{Q}_{n}\right)=\sum_{C \in \mathcal{D}} \lambda_{1}^{k_{1}^{C}} \cdots \lambda_{n}^{k_{n}^{C}} \operatorname{Vol}_{n}(C)
$$

where for each $C \in \mathcal{D},\left(k_{1}^{C}, \ldots, k_{n}^{C}\right)$ denotes the type of $C$, i.e., $\operatorname{Vol}_{n}\left(\lambda_{1} \mathcal{Q}_{1}+\right.$ $\left.\cdots+\lambda_{n} \mathcal{Q}_{n}\right)$ is a homogeneous polynomial of degree $n$ in $\left(\lambda_{1}, \ldots, \lambda_{n}\right)$.

Proof. By Proposition $4, \boldsymbol{\lambda} \circ \mathcal{D}=\{\boldsymbol{\lambda} \circ C \mid C \in \mathcal{D}\}$ is also a fine mixed subdivision of $\boldsymbol{\lambda} \circ \mathcal{S}$. Therefore the volume $\operatorname{Vol}_{n}\left(\lambda_{1} \mathcal{Q}_{1}+\cdots+\lambda_{n} \mathcal{Q}_{n}\right)$ is the sum of the volumes of the cells in $\boldsymbol{\lambda} \circ \mathcal{D}$, and by applying Proposition 11 formula (11) holds.

Also note that for each cell $C \in \mathcal{D}$ of type $\left(k_{1}^{C}, \ldots, k_{n}^{C}\right)$, condition (d1) in the definition of fine mixed subdivision requires that $k_{1}^{C}+\cdots+k_{n}^{C}$ must be exactly $n$. Therefore each term in the above polynomial expression has a (total) degree of $n$ in $\left(\lambda_{1}, \ldots, \lambda_{n}\right)$, and hence it is homogeneous.

It follows that in the homogeneous polynomial $\operatorname{Vol}_{n}\left(\lambda_{1} \mathcal{Q}_{1}+\cdots+\lambda_{n} \mathcal{Q}_{n}\right)$, the contribution of each cell in $\mathcal{D}$ is a monomial term in $\lambda_{1}, \ldots, \lambda_{n}$. Its coefficient is determined by the volume of the cell, and its exponents are given by the type of the cell. Recall that, by definition, the mixed volume $\mathcal{M}\left(\mathcal{Q}_{1}, \ldots, \mathcal{Q}_{n}\right)$ is the coefficient of $\lambda_{1} \times \cdots \times \lambda_{n}$ in $\operatorname{Vol}_{n}\left(\lambda_{1} \mathcal{Q}_{1}+\cdots+\right.$ $\left.\lambda_{n} \mathcal{Q}_{n}\right)$, so, from (11),

$$
\mathcal{M}\left(\mathcal{Q}_{1}, \ldots, \mathcal{Q}_{n}\right)=\sum_{\substack{C \in \mathcal{D} \\ \operatorname{type}(C)=(1, \ldots, 1)}} \operatorname{Vol}_{n}(C) .
$$

Moreover, since $\mathcal{D}$ is a fine mixed subdivision, each cell $C \in \mathcal{D}$ of type $(1, \ldots, 1)$ is necessarily of the form $C=\left(\left\{\boldsymbol{c}_{1}, \boldsymbol{c}_{1}^{\prime}\right\}, \ldots,\left\{\boldsymbol{c}_{n}, \boldsymbol{c}_{n}^{\prime}\right\}\right)$ where $\left\{\boldsymbol{c}_{i}, \boldsymbol{c}_{i}^{\prime}\right\}$ $\subseteq \mathcal{S}_{i}$. So each $V_{i}(C)$, defined in (5), consists of a single column, and

$$
V(C)=\left[\begin{array}{lll}
\boldsymbol{c}_{1}-\boldsymbol{c}_{1}^{\prime} & \cdots & \boldsymbol{c}_{n}-\boldsymbol{c}_{n}^{\prime}
\end{array}\right]
$$

as defined in (6). Accordingly, from (10),

$$
\text { (13) } \mathcal{M}\left(\mathcal{Q}_{1}, \ldots, \mathcal{Q}_{n}\right)=\sum_{\left(\left\{\boldsymbol{c}_{1}, \boldsymbol{c}_{1}^{\prime}\right\}, \ldots,\left\{\boldsymbol{c}_{n}, \boldsymbol{c}_{n}^{\prime}\right\}\right) \in \mathcal{D}}\left|\operatorname{det}\left[\boldsymbol{c}_{1}-\boldsymbol{c}_{1}^{\prime} \cdots \boldsymbol{c}_{n}-\boldsymbol{c}_{n}^{\prime}\right]\right| .
$$


Remark 13. Formula (12) reveals an important property: The mixed volume is indeed nonnegative, which is not immediately obvious from its definition. Moreover, when $\mathcal{S}$ consists of integral lattice points the mixed volume $\mathcal{M}(\mathcal{Q})$ is an integer and in fact counts the generic number of complex roots of a polynomial system as in the BeRnshtein's theorem [1, 16, 23, 27.

The above construction suggests a clear strategy for computing mixed volume: With a fine mixed subdivision of $\mathcal{S}=\left(\mathcal{S}_{1}, \ldots, \mathcal{S}_{n}\right)$, one may systematically enumerate all the mixed cells of type $(1, \ldots, 1)$, and then the sum of the volume of all these cells as given in 10 is precisely the mixed volume $\mathcal{M}\left(\mathcal{Q}_{1}, \ldots, \mathcal{Q}_{n}\right)$.

\section{Mixed subdivisions induced by generic lifting}

We now discuss a constructive procedure, developed in [2, 16], with which a fine mixed subdivision of $\mathcal{S}=\left(\mathcal{S}_{1}, \ldots, \mathcal{S}_{n}\right)$ can be found.

For each $i=1, \ldots, n$, let $\boldsymbol{\omega}_{i}: \mathcal{S}_{i} \rightarrow \mathbb{R}$ be a function that assigns each point in $\mathcal{S}_{i}$ a real number. The function $\boldsymbol{\omega}:=\left(\boldsymbol{\omega}_{1}, \ldots, \boldsymbol{\omega}_{n}\right)$ is known as a lifting function on $\mathcal{S}=\left(\mathcal{S}_{1}, \ldots, \mathcal{S}_{n}\right)$. For each $i=1, \ldots, n$, consider the "lifted" versions of $\mathcal{S}_{i}$ and conv $\mathcal{S}$ given by

$$
\hat{\mathcal{S}}_{i}:=\left\{\left(\boldsymbol{a}, \boldsymbol{\omega}_{i}(\boldsymbol{a})\right): \boldsymbol{a} \in \mathcal{S}_{i}\right\} \quad \text { and } \quad \operatorname{conv} \hat{\mathcal{S}}:=\hat{\mathcal{S}}_{1}+\cdots+\hat{\mathcal{S}}_{n}
$$

respectively. Let $\pi: \mathbb{R}^{n+1} \rightarrow \mathbb{R}^{n}$ be the projection by erasing the last coordinate, so $\pi\left(\hat{\mathcal{S}}_{i}\right)=\mathcal{S}_{i}$ for each $i=1, \ldots, n$, and $\pi(\operatorname{conv} \hat{\mathcal{S}})=\operatorname{conv} \mathcal{S}$.

Consider the polytope conv $\hat{\mathcal{S}}$, now in $\mathbb{R}^{n+1}$. We are interested in its "lower hull" with respect to the projection $\pi$ : A vector $\hat{\boldsymbol{\alpha}} \in \mathbb{R}^{n+1}$ is said to be upward pointing if it has positive last coordinate. Without loss of generality, we may assume the last coordinate of an upward pointing $\hat{\boldsymbol{\alpha}}$ is 1 , that is, $\hat{\boldsymbol{\alpha}}=\left(\alpha_{1}, \ldots, \alpha_{n}, 1\right) \in \mathbb{R}^{n+1}$. A face $\hat{F}$ of conv $\hat{\mathcal{S}}$ is called a lower face if its inner normal is upward pointing, namely, there exists an $\hat{\boldsymbol{\alpha}}=$ $\left(\alpha_{1}, \ldots, \alpha_{n}, 1\right) \in \mathbb{R}^{n+1}$ such that $\hat{F}=(\operatorname{conv} \hat{\mathcal{S}})_{\hat{\alpha}}$. It is important to note that for a lower face $\hat{F}$ of conv $\hat{\mathcal{S}}$, by linearity,

$$
\hat{F}=(\operatorname{conv} \hat{\mathcal{S}})_{\hat{\boldsymbol{\alpha}}}=\left(\operatorname{conv} \hat{\mathcal{S}}_{1}\right)_{\hat{\boldsymbol{\alpha}}}+\cdots+\left(\operatorname{conv} \hat{\mathcal{S}}_{n}\right)_{\hat{\boldsymbol{\alpha}}}
$$

for some upward pointing normal $\hat{\boldsymbol{\alpha}}$. In other words, a lower face of conv $\hat{\mathcal{S}}$ is necessarily a Minkowski sum of $n$ lower faces of conv $\hat{\mathcal{S}}_{1}, \ldots$, conv $\hat{\mathcal{S}}_{n}$ respectively sharing a common inner normal of the form $\hat{\boldsymbol{\alpha}}=\left(\alpha_{1}, \ldots, \alpha_{n}, 1\right)$. The lower hull of conv $\hat{\mathcal{S}}$ is the collection of all of its $n$-dimensional lower faces. 
We shall impose a "genericity" condition on the lifting function. To facilitate the discussion, the following notation will be used: Fix any $i \in$ $\{1, \ldots, n\}$ and a subset $X_{i}=\left\{\boldsymbol{x}_{i, 1}, \ldots, \boldsymbol{x}_{i, m_{i}}\right\} \subseteq \mathcal{S}_{i}$, containing $m_{i}$ points for some $m_{i}>0$, define

$$
V\left(X_{i}\right)=\left(\begin{array}{c}
\boldsymbol{x}_{i, 2}^{\top}-\boldsymbol{x}_{i, 1}^{\top} \\
\boldsymbol{x}_{i, 3}^{\dagger}-\boldsymbol{x}_{i, 1}^{\top} \\
\vdots \\
\boldsymbol{x}_{i, m_{i}}^{\top}-\boldsymbol{x}_{i, 1}^{\top}
\end{array}\right) \quad \text { and } \quad \Omega\left(X_{i}\right)=\left(\begin{array}{c}
\boldsymbol{\omega}_{i}\left(\boldsymbol{x}_{i, 1}\right)-\boldsymbol{\omega}_{i}\left(\boldsymbol{x}_{i, 2}\right) \\
\boldsymbol{\omega}_{i}\left(\boldsymbol{x}_{i, 1}\right)-\boldsymbol{\omega}_{i}\left(\boldsymbol{x}_{i, 2}\right) \\
\vdots \\
\boldsymbol{\omega}_{i}\left(\boldsymbol{x}_{i, 1}\right)-\boldsymbol{\omega}_{i}\left(\boldsymbol{x}_{i, m_{i}}\right)
\end{array}\right)
$$

Definition 14. A lifting function $\boldsymbol{\omega}=\left(\boldsymbol{\omega}_{1}, \ldots, \boldsymbol{\omega}_{n}\right)$ for $\mathcal{S}=\left(\mathcal{S}_{1}, \ldots, \mathcal{S}_{n}\right)$ is said to be generic if for any choice of $n$ (possibly empty) subsets $X_{i}=$ $\left\{\boldsymbol{x}_{i, 1}, \ldots, \boldsymbol{x}_{i, m_{i}}\right\} \subseteq \mathcal{S}_{i}$ for $i=1, \ldots, n$ with $m_{i} \geq 0$ the linear system

$$
\left(\begin{array}{c}
V\left(X_{1}\right) \\
V\left(X_{2}\right) \\
\vdots \\
V\left(X_{n}\right)
\end{array}\right) \cdot \boldsymbol{\alpha}=\left(\begin{array}{c}
\Omega\left(X_{1}\right) \\
\Omega\left(X_{2}\right) \\
\vdots \\
\Omega\left(X_{n}\right)
\end{array}\right)
$$

in $\boldsymbol{\alpha}$ has an isolated solution only when the rank of the matrix on the left equals the number of its rows. Note that if the subset $X_{i}$ is empty, the blocks $V\left(X_{i}\right)$ and $\Omega\left(X_{i}\right)$ will not appear in the above equation.

Remark 15. One important observation is that almost all liftings are generic in the sense of Definition 14, justifying the choice of the terminology. More precisely, for each $i=1, \ldots, n$, we can identify $\boldsymbol{\omega}_{i}: \mathcal{S}_{i} \rightarrow \mathbb{R}$ with its images and consider $\boldsymbol{\omega}_{i}$ as an element in $\mathbb{R}^{N_{i}}$ where $N_{i}=\# \mathcal{S}_{i}$. Similarly, we consider $\boldsymbol{\omega}=\left(\boldsymbol{\omega}_{1}, \ldots, \boldsymbol{\omega}_{n}\right)$ as an element in $\mathbb{R}^{N}$ where $N=N_{1}+\cdots+N_{n}$. If $\boldsymbol{\omega}$ is not generic, then there exists a choice of $n$ (possibly empty) subsets $\left\{\boldsymbol{x}_{i, 1}, \ldots, \boldsymbol{x}_{i, m_{i}}\right\} \subseteq \mathcal{S}_{i}$ for $i=1, \ldots, n$ with $m_{i}>0$ for which the rank of the matrix on the left hand side of the linear system 15 is less than the number of its rows but the system has a solution. This condition forces $\boldsymbol{\omega}$ to be in an affine subspace of lower dimension. Since there are only finitely many ways of choosing subsets of $\mathcal{S}_{1}, \ldots, \mathcal{S}_{n}$, the set of non-generic liftings is thus contained in a finite union of lower dimensional affine subspaces of $\mathbb{R}^{N}$ determined by the points in $\mathcal{S}$. This set is necessarily of measure zero. Indeed, this set is closed and nowhere dense. This is of great practical importance: one can choose a lifting at random, then the probability of choosing a non-generic one is zero. 
Proposition 16 (Induced fine mixed subdivision). Let $\boldsymbol{\omega}=(\boldsymbol{\omega}, \ldots$, $\boldsymbol{\omega}_{n}$ ) be a generic lifting for $\mathcal{S}=\left(\mathcal{S}_{1}, \ldots, \mathcal{S}_{n}\right)$, and let $\hat{\mathcal{D}}_{\boldsymbol{\omega}}$ be the collection of all $\hat{C}=\left(\hat{C}_{1}, \ldots, \hat{C}_{n}\right)$ with $\hat{C}_{i} \subseteq \hat{\mathcal{S}}_{i}$ for each $i=1, \ldots, n$ such that

1) $\operatorname{conv} \hat{C}_{i}$ is a face of conv $\hat{\mathcal{S}}_{i}$ for each $i=1, \ldots, n$;

2) The $n$ faces conv $\hat{C}_{i}$ of conv $\hat{\mathcal{S}}_{1}, \ldots$, conv $\hat{\mathcal{S}}_{n}$ respectively share a common inner normal of the form $\hat{\boldsymbol{\alpha}}=\left(\alpha_{1}, \ldots, \alpha_{n}, 1\right)$; and

3) $\operatorname{dim}\left(\operatorname{conv}(\hat{C})_{1}+\cdots+\operatorname{conv}(\hat{C})_{n}\right)=n$.

Then the projection

$$
\mathcal{D}_{\boldsymbol{\omega}}=\left\{\left(\pi\left(\hat{C}_{1}\right), \ldots, \pi\left(\hat{C}_{n}\right)\right) \mid \hat{C}=\left(\hat{C}_{1}, \ldots, \hat{C}_{n}\right) \in \hat{\mathcal{D}}_{\boldsymbol{\omega}}\right\}
$$

form a fine mixed subdivision of $\mathcal{S}=\left(\mathcal{S}_{1}, \ldots, \mathcal{S}_{n}\right)$, called the fine mixed subdivision induced by the lifting function $\omega=(\omega, \ldots, \omega)$.

Proof. We shall prove $\mathcal{D}_{\boldsymbol{\omega}}$ satisfies the conditions (a),(b),(c),(d1),(d2) and (e) in Definitions 1, 2, and 3 of a fine mixed subdivision of $\mathcal{S}=\left(\mathcal{S}_{1}, \ldots, \mathcal{S}_{n}\right)$.

(a) We need to show that for any $C=\left(C_{1}, \ldots, C_{n}\right) \in \mathcal{D}_{\boldsymbol{\omega}}, \operatorname{dim}(\operatorname{conv} C)=$ $n$. Since conv $\hat{C}$ is a lower facet of conv $\hat{\mathcal{S}}$, there exists a vector $\hat{\boldsymbol{\alpha}}=$ $(\boldsymbol{\alpha}, 1) \in \mathbb{R}^{n+1} \operatorname{such}$ that $\operatorname{conv} \hat{C}=(\operatorname{conv} \hat{\mathcal{S}})_{\hat{\boldsymbol{\alpha}}}$. Now suppose $\operatorname{dim}(\operatorname{conv} C)$ $<n$, then $\operatorname{conv} C$ must lie in some hyperplane in $\mathbb{R}^{n}$, that is, there exists a vector $\boldsymbol{\beta} \in \mathbb{R}^{n}$ such that $\operatorname{conv} C \subseteq\left\{\boldsymbol{x} \in \mathbb{R}^{n}:\langle\boldsymbol{x}, \boldsymbol{\beta}\rangle=h\right\}$ for some $h \in \mathbb{R}$. Then for any $\hat{\boldsymbol{x}}=\left(x_{1}, \ldots, x_{n+1}\right) \in \operatorname{conv} \hat{C}, \boldsymbol{x}:=\pi\left(\left(x_{1}, \ldots\right.\right.$, $\left.\left.x_{n+1}\right)\right) \in \operatorname{conv} C$, and so $\langle\hat{\boldsymbol{x}},(\boldsymbol{\beta}, 0)\rangle=\langle\boldsymbol{x}, \boldsymbol{\beta}\rangle+x_{n+1} \cdot 0=h$. Thus conv $\hat{C}$ satisfies two independent linear equations in $\mathbb{R}^{n+1}$ :

$$
\begin{aligned}
\langle\hat{\boldsymbol{x}},(\boldsymbol{\alpha}, 1)\rangle & =\operatorname{ht}_{\hat{\boldsymbol{\alpha}}}(\operatorname{conv} \hat{C}) \\
\langle\hat{\boldsymbol{x}},(\boldsymbol{\beta}, 0)\rangle & =h,
\end{aligned}
$$

and its dimension is therefore at most $n+1-2=n-1$, contradicting to the fact that conv $\hat{C}$ is a facet of an $(n+1)$-dimensional polytope conv $\hat{\mathcal{S}}$ by construction. So conv $C$ must be $n$-dimensional.

(b) For any two distinct cells $A, B \in \mathcal{D}_{\boldsymbol{\omega}}$, if $F=(\operatorname{conv} A) \cap(\operatorname{conv} B) \subset \mathbb{R}^{n}$ is nonempty, we need to show that $F$ is a common face of both conv $A$ and conv $B$. Let $\hat{F}:=\operatorname{conv} \hat{A} \cap \operatorname{conv} \hat{B} \subset \mathbb{R}^{n+1}$. Clearly, $F$ is a projection of $\hat{F}$ onto $\left\{\boldsymbol{e}_{n+1}\right\}^{\perp}$ where $\boldsymbol{e}_{n+1}=(0, \ldots, 0,1)^{\top} \in \mathbb{R}^{n+1}$. By definition, conv $\hat{A}$ and conv $\hat{B}$ are lower facets of conv $\hat{\mathcal{S}}$, so there exist vectors $\hat{\boldsymbol{\alpha}}=(\boldsymbol{\alpha}, 1)$ and $\hat{\boldsymbol{\beta}}=(\boldsymbol{\beta}, 1)$ in $\mathbb{R}^{n+1}$ such that $\operatorname{conv} \hat{A}=(\operatorname{conv} \hat{\mathcal{S}})_{\hat{\boldsymbol{\alpha}}}$ and $\operatorname{conv} \hat{B}=(\operatorname{conv} \hat{\mathcal{S}})_{\hat{\boldsymbol{\beta}}}$. Let $h_{1}=\mathrm{ht}_{\hat{\boldsymbol{\alpha}}}(\operatorname{conv} \hat{\mathcal{S}})$ and $h_{2}=\mathrm{ht}_{\hat{\boldsymbol{\beta}}}(\operatorname{conv} \hat{\mathcal{S}})$. 
For any $\boldsymbol{x} \in F$, there exists $t \in \mathbb{R}$ such that $\hat{\boldsymbol{x}}=(\boldsymbol{x}, t) \in \hat{F}=\operatorname{conv} \hat{A}$ $\cap \operatorname{conv} \hat{B}$. Thus

$$
h_{1}=\langle\hat{\boldsymbol{x}}, \hat{\boldsymbol{\alpha}}\rangle=\langle\boldsymbol{x}, \alpha\rangle+t, \quad h_{2}=\langle\hat{\boldsymbol{x}}, \hat{\boldsymbol{\beta}}\rangle=\langle\boldsymbol{x}, \boldsymbol{\beta}\rangle+t
$$

Taking the difference of these two equations yields

$$
\langle\boldsymbol{x}, \boldsymbol{\beta}-\boldsymbol{\alpha}\rangle=h_{2}-h_{1}
$$

which holds for any $\boldsymbol{x} \in F$. For any $\boldsymbol{x} \in \operatorname{conv} A \backslash F$, there exists $s \in \mathbb{R}$ such that $\hat{\boldsymbol{x}}=(\boldsymbol{x}, s)$ is in conv $\hat{A}$ but not in conv $\hat{B}$. Consequently,

$$
h_{1}=\langle\hat{\boldsymbol{x}}, \hat{\boldsymbol{\alpha}}\rangle=\langle\boldsymbol{x}, \boldsymbol{\alpha}\rangle+s, \quad h_{2}<\langle\hat{\boldsymbol{x}}, \hat{\boldsymbol{\beta}}\rangle=\langle\boldsymbol{x}, \boldsymbol{\beta}\rangle+s
$$

Again, taking the difference of these two equations results in

$$
\langle\boldsymbol{x}, \boldsymbol{\beta}-\boldsymbol{\alpha}\rangle>h_{2}-h_{1}
$$

Therefore the linear functional $\langle\bullet, \boldsymbol{\beta}-\boldsymbol{\alpha}\rangle$ minimizes over $\operatorname{conv} A$ at $F$, and hence $F$ is a face of conv $A$. By the same argument, functional $\langle\bullet, \boldsymbol{\alpha}-\boldsymbol{\beta}\rangle$ minimizes over conv $B$ at $F$, and hence $F$ is a face of conv $B$ as well. Therefore $F$ is a common face of $\operatorname{conv} A$ and $\operatorname{conv} B$.

(c) We need to show $\bigcup_{C \in \mathcal{D}_{\omega}} \operatorname{conv} C=\operatorname{conv} \mathcal{S}$. Clearly, conv $C \subseteq \operatorname{conv} \mathcal{S}$ for each $C \in \mathcal{D}_{\boldsymbol{\omega}}$, so $\bigcup_{C \in \mathcal{D}_{\boldsymbol{\omega}}} \operatorname{conv} C \subseteq$ conv $S$. For the other direction, fix any $\boldsymbol{q} \in \operatorname{conv} \mathcal{S}$, let $W=(\{\boldsymbol{q}\} \times \mathbb{R}) \cap \operatorname{conv} \hat{\mathcal{S}}$, which is a closed set. Since conv $\hat{\mathcal{S}}$ is a polytope, $W$ must be bounded and hence compact, so $\ell=\min \{t \in \mathbb{R}:(\boldsymbol{q}, t) \in \operatorname{conv} \hat{\mathcal{S}}\}$ exists. Let $\hat{\boldsymbol{q}}=(\boldsymbol{q}, \ell)$, then $\hat{\boldsymbol{q}} \in \partial(\operatorname{conv} \hat{\mathcal{S}})$, so there must be an $n$-dimensional facet $\hat{F}$ of $\operatorname{conv} \hat{\mathcal{S}}$ containing $\hat{\boldsymbol{q}}$. We shall show that $\hat{F}$ is indeed a lower facet.

First, if $\operatorname{dim}(\operatorname{conv} \hat{\mathcal{S}})=n$, then all faces are lower faces. Otherwise, let $\hat{\boldsymbol{\alpha}}=\left(\alpha_{1}, \ldots, \alpha_{n+1}\right)$ be the inner normal of $\hat{F}$. For $\epsilon>0$ small enough so that $(\boldsymbol{q}, \ell+\epsilon) \in \operatorname{conv} \hat{\mathcal{S}}$, then

$$
\langle(\boldsymbol{q}, \ell+\epsilon), \hat{\boldsymbol{\alpha}}\rangle=\langle\hat{\boldsymbol{q}}, \hat{\boldsymbol{\alpha}}\rangle+\epsilon \cdot \alpha_{n+1}>\langle\hat{\boldsymbol{q}}, \hat{\boldsymbol{\alpha}}\rangle
$$

since $\langle\bullet, \hat{\boldsymbol{\alpha}}\rangle$ minimizes on conv $\hat{\mathcal{S}}$ at $\hat{F}$. This implies $\alpha_{n+1}>0$. Therefore $\hat{F}$ is a lower facet, and so there is a cell $C \in \mathcal{D}_{\boldsymbol{\omega}}$ such that conv $\hat{C}=$ $\hat{F}$; in particular, $\boldsymbol{q} \in \operatorname{conv} C$. Therefore $\bigcup_{C \in \mathcal{D}_{\omega}} \operatorname{conv} C \supseteq \operatorname{conv} \mathcal{S}$. 
(d2) Let cells $A=\left(A_{1}, \ldots, A_{n}\right), B=\left(B_{1}, \ldots, B_{n}\right) \in \mathcal{D}_{\boldsymbol{\omega}}$ with $F:=(\operatorname{conv} A)$ $\cap(\operatorname{conv} B)$ being nonempty. We shall show

$$
\operatorname{conv} A \cap \operatorname{conv} B=\sum_{i=1}^{n}\left(\operatorname{conv} A_{i}\right) \cap\left(\operatorname{conv} B_{i}\right)
$$

One direction of the containment is obvious: since for any collection of $\boldsymbol{x}_{i} \in\left(\operatorname{conv} A_{i}\right) \cap\left(\operatorname{conv} B_{i}\right)$ for $i=1, \ldots, n, \boldsymbol{x}:=\boldsymbol{x}_{1}+\cdots+\boldsymbol{x}_{n}$ is in both conv $A$ and conv $B$ and hence $\sum_{i=1}^{n}\left(\operatorname{conv} A_{i}\right) \cap\left(\operatorname{conv} B_{i}\right) \subseteq$ $(\operatorname{conv} A) \cap(\operatorname{conv} B)$.

For the other direction, since conv $\hat{B}$ is a lower facet of conv $\hat{\mathcal{S}}$, let $\hat{\boldsymbol{\beta}}=(\boldsymbol{\beta}, 1) \in \mathbb{R}^{n+1}$ be its inner normal, then

$$
\operatorname{conv} \hat{B}=(\operatorname{conv} \hat{\mathcal{S}})_{\hat{\boldsymbol{\beta}}}=\sum_{i=1}^{n}\left(\operatorname{conv} \hat{\mathcal{S}}_{i}\right)_{\hat{\boldsymbol{\beta}}} \text { with }\left(\hat{\mathcal{S}}_{i}\right)_{\hat{\boldsymbol{\beta}}}=\operatorname{conv} \hat{B}_{i}
$$

Picking any $\boldsymbol{a} \in F$, consider its corresponding $\hat{\boldsymbol{a}} \in(\operatorname{conv} \hat{A}) \cap(\operatorname{conv} \hat{B})$. Then $\hat{\boldsymbol{a}}=\hat{\boldsymbol{a}}_{1}+\cdots+\hat{\boldsymbol{a}}_{n}$ for some $\hat{\boldsymbol{a}}_{i} \in \operatorname{conv} \hat{A}_{i}$. Moreover, $\hat{\boldsymbol{a}} \in \operatorname{conv} \hat{B}$ implies

$$
\langle\hat{\boldsymbol{a}}, \hat{\boldsymbol{\beta}}\rangle=\sum_{i=1}^{n}\left\langle\hat{\boldsymbol{a}}_{i}, \hat{\boldsymbol{\beta}}\right\rangle=\mathrm{ht}_{\hat{\boldsymbol{\beta}}}(\operatorname{conv} \hat{\mathcal{S}})=\sum_{i=1}^{n} \mathrm{ht}_{\hat{\boldsymbol{\beta}}}\left(\operatorname{conv} \hat{\mathcal{S}}_{i}\right)
$$

But for each $i=1, \ldots, n,\left\langle\hat{\boldsymbol{a}}_{i}, \hat{\boldsymbol{\beta}}\right\rangle \geq \mathrm{ht}_{\hat{\boldsymbol{\beta}}}\left(\operatorname{conv} \hat{\mathcal{S}}_{i}\right)$. Therefore $\left\langle\hat{\boldsymbol{a}}_{i}, \hat{\boldsymbol{\beta}}\right\rangle=$ $\mathrm{ht}_{\hat{\boldsymbol{\beta}}}\left(\operatorname{conv} \hat{\mathcal{S}}_{i}\right)$ for $i=1, \ldots, n$, and hence each $\hat{\boldsymbol{a}}_{i} \in\left(\operatorname{conv} \hat{\mathcal{S}}_{i}\right)_{\hat{\boldsymbol{\beta}}}=\operatorname{conv} \hat{B}_{i}$. Consequently, $\hat{\boldsymbol{a}}_{i} \in\left(\operatorname{conv} \hat{A}_{i}\right) \cap\left(\operatorname{conv} \hat{B}_{i}\right)$ for each $i=1, \ldots, n$, thus $\hat{\boldsymbol{a}}=\sum_{i=1}^{n} \hat{\boldsymbol{a}}_{i} \in \sum_{i=1}^{n}\left(\operatorname{conv} \hat{A}_{i}\right) \cap\left(\operatorname{conv} \hat{B}_{i}\right)$. Projection via $\pi$ yields $\boldsymbol{a} \in$ $\sum_{i=1}^{n}\left(\operatorname{conv} A_{i}\right) \cap\left(\operatorname{conv} B_{i}\right)$ and therefore

$$
(\operatorname{conv} A) \cap(\operatorname{conv} B) \subseteq \sum_{i=1}^{n}\left(\operatorname{conv} A_{i}\right) \cap\left(\operatorname{conv} B_{i}\right)
$$

(e) For a cell $C=\left(C_{1}, \ldots, C_{n}\right) \in \mathcal{D}_{\boldsymbol{\omega}}$, we fix an $i$ in $\{1, \ldots, n\}$ and consider $C_{i}$ only. Let $m_{i}=\# C_{i}-1$, then $C_{i}=\left\{\boldsymbol{c}_{i, 0}, \ldots, \boldsymbol{c}_{i, m_{i}}\right\} \subset \mathcal{S}_{i}$. Let $\hat{\boldsymbol{\alpha}}=$ $(\boldsymbol{\alpha}, 1)$ be an inner normal of $\operatorname{conv} \hat{C}$ in $\operatorname{conv} \hat{\mathcal{S}}$. Then

$$
\left\langle\hat{\boldsymbol{c}}_{i, j}, \hat{\boldsymbol{\alpha}}\right\rangle=\left\langle\left(\boldsymbol{c}_{i, j}, \boldsymbol{\omega}_{i}\left(\boldsymbol{c}_{i, j}\right)\right),(\boldsymbol{\alpha}, 1)\right\rangle=\left\langle\boldsymbol{c}_{i, j}, \boldsymbol{\alpha}\right\rangle+\boldsymbol{\omega}_{i}\left(\boldsymbol{c}_{i, j}\right)=\mathrm{ht}_{\hat{\boldsymbol{\alpha}}}\left(\operatorname{conv} \hat{\mathcal{S}}_{i}\right)
$$


for each $j=0, \ldots, m_{i}$. This can be written as

$$
\left(\begin{array}{c}
\boldsymbol{c}_{i, 1}^{\top}-\boldsymbol{c}_{i, 0}^{\top} \\
\vdots \\
\boldsymbol{c}_{i, m_{i}}^{\top}-\boldsymbol{c}_{i, 0}^{\top}
\end{array}\right) \cdot \boldsymbol{\alpha}=\left(\begin{array}{c}
\boldsymbol{\omega}_{i}\left(\boldsymbol{c}_{i, 0}\right)-\boldsymbol{\omega}_{i}\left(\boldsymbol{c}_{i, 1}\right) \\
\vdots \\
\boldsymbol{\omega}_{i}\left(\boldsymbol{c}_{i, 0}\right)-\boldsymbol{\omega}_{i}\left(\boldsymbol{c}_{i, m_{i}}\right)
\end{array}\right)
$$

Suppose conv $C_{i}$ is not a simplex, then those $m_{i}$ points $\boldsymbol{c}_{i, 0}, \ldots, \boldsymbol{c}_{i, m_{i}}$ must be affinely dependent. It follows that matrix on the left hand side of the above equation is of rank less than $m_{i}$. This contradicts to the genericity of the lifting (Definition 14).

(d1) Fix a cell $C=\left(C_{1}, \ldots, C_{n}\right) \in \mathcal{D}_{\boldsymbol{\omega}}$ with $C_{i}=\left\{\boldsymbol{c}_{0}, \ldots, \boldsymbol{c}_{m_{i}}\right\}$ where $m_{i}=$ $\# C_{i}-1$ for each $i=1, \ldots, n$. Assume condition (e) is already satisfied, that is, for each $i=1, \ldots, n, \operatorname{conv} C_{i}$ is a simplex of dimension $m_{i}$. Then it is enough to show that $m_{1}+\cdots+m_{n}=n$. Let $\hat{\boldsymbol{\alpha}}=(\boldsymbol{\alpha}, 1)$ be an inner normal of conv $\hat{C}$ in conv $\hat{\mathcal{S}}$. Then $\hat{\boldsymbol{\alpha}}$ satisfies 16 for each $i=1, \ldots, n$ and $j=0, \ldots, m_{i}$. Similar to (17), these equations can be combined into

$$
\left(\begin{array}{c}
\boldsymbol{c}_{1,1}^{\top}-\boldsymbol{c}_{1,0}^{\top} \\
\vdots \\
\boldsymbol{c}_{1, m_{1}}^{\top}-\boldsymbol{c}_{1,0}^{\top} \\
\vdots \\
\boldsymbol{c}_{n, 1}^{\top}-\boldsymbol{c}_{n, 0}^{\top} \\
\vdots \\
\boldsymbol{c}_{n, m_{1}}^{\top}-\boldsymbol{c}_{n, 0}^{\top}
\end{array}\right) \cdot \boldsymbol{\alpha}=\left(\begin{array}{c}
\boldsymbol{\omega}_{1}\left(\boldsymbol{c}_{1,0}\right)-\boldsymbol{\omega}_{1}\left(\boldsymbol{c}_{1,1}\right) \\
\vdots \\
\boldsymbol{\omega}_{1}\left(\boldsymbol{c}_{1,0}\right)-\boldsymbol{\omega}_{1}\left(\boldsymbol{c}_{1, m_{1}}\right) \\
\vdots \\
\boldsymbol{\omega}_{n}\left(\boldsymbol{c}_{n, 0}\right)-\boldsymbol{\omega}_{n}\left(\boldsymbol{c}_{n, 1}\right) \\
\vdots \\
\boldsymbol{\omega}_{n}\left(\boldsymbol{c}_{n, 0}\right)-\boldsymbol{\omega}_{n}\left(\boldsymbol{c}_{n, m_{i}}\right)
\end{array}\right)
$$

Here, the matrix on the left hand side of the above equation is of the size $\left(m_{1}+\cdots+m_{n}\right) \times n$. By the genericity assumption (Definition 14 the rank of this matrix must be $m_{1}+\cdots+m_{n}$. Therefore $m_{1}+\cdots+$ $m_{n} \leq n$.

Recall that condition (a) asserts $\operatorname{dim}(\operatorname{conv} C)=n$. Since $m_{1}+\cdots+$ $m_{n}=\operatorname{dim}\left(\operatorname{conv} C_{1}\right)+\cdots+\operatorname{dim}\left(\operatorname{conv} C_{n}\right) \geq n$, thus $m_{1}+\cdots+m_{n}=n$.

Therefore $\mathcal{D}_{\boldsymbol{\omega}}$ satisfies condition (a)-(e) in Definition 1, 2, and 3 , and hence it is a fine mixed subdivision for $\mathcal{S}$. 


\section{The semi-mixed case}

The $n$-tuple $\mathcal{S}=\left(\mathcal{S}_{1}, \ldots, \mathcal{S}_{n}\right)$ is called semi-mixed of type $\left(r_{1}, \ldots, r_{m}\right)$ when $\mathcal{S}_{i}$ 's are not all distinct, but they are equal within $m$ blocks of sizes $r_{1}, \ldots, r_{m}$, i.e., there are $m$ sets $\mathcal{S}^{(1)}, \ldots, \mathcal{S}^{(r)} \subset \mathbb{R}^{n}$ such that $\mathcal{S}^{(i)}=\mathcal{S}_{i, 1}=$ $\cdots=\mathcal{S}_{i, r_{i}}$ where

$$
\mathcal{S}_{i, j} \in\left\{\mathcal{S}_{1}, \cdots, \mathcal{S}_{n}\right\} \quad \text { for } \quad 1 \leq i \leq m, \quad 1 \leq j \leq r_{i},
$$

and $r_{1}+\cdots+r_{m}=n$. $\mathcal{S}$ is called unmixed if $m=1$, i.e., when all the $\mathcal{S}_{i}^{\prime} s$ are identical. $\mathcal{S}$ is called fully mixed if $m=n$, i.e., when the $\mathcal{S}_{i}^{\prime} s$ are distinct. We abbreviate $\mathcal{S}=\left(\mathcal{S}^{(1)}, r_{1} ; \mathcal{S}^{(2)}, r_{2} ; \cdots ; \mathcal{S}^{(m)}, r_{m}\right)$, and $\mathcal{Q}=$ $\left(\mathcal{Q}^{(1)}, r_{1} ; \mathcal{Q}^{(2)}, r_{2} ; \cdots ; \mathcal{Q}^{(m)}, r_{m}\right)$, with $\mathcal{Q}^{(i)}=\operatorname{conv} \mathcal{S}^{(i)}$ for $i=1, \ldots, m$.

To calculate the mixed volume of a semi-mixed system $\mathcal{S}=\left(\mathcal{S}^{(1)}, r_{1}\right.$; $\left.\mathcal{S}^{(2)}, r_{2} ; \cdots ; \mathcal{S}^{(m)}, r_{m}\right)$, one may, of course, follow the standard procedure described in $\$$ without paying a special attention to its semi-mixed structure. However, when this special structure is taken into account, a revised procedure may be developed with a great reduction in the amount of computation, especially when $\mathcal{S}$ is unmixed such as the nine-point path synthesis problem for four-bar linkages [30] in mechanical design.

Now, the mixed volume $\mathcal{M}\left(\mathcal{Q}^{(1)}, r_{1} ; \mathcal{Q}^{(2)}, r_{2} ; \cdots ; \mathcal{Q}^{(m)}, r_{m}\right)$ is, by definition, the coefficient of $\prod_{j=1}^{r_{1}} \lambda_{1, j} \cdots \prod_{j=1}^{r_{m}} \lambda_{m, j}$ in the expansion of the homogeneous polynomial

$$
\operatorname{Vol}_{n}\left(\sum_{i=1}^{m} \sum_{j=1}^{r_{i}} \lambda_{i j} \mathcal{Q}^{(i)}\right)=\operatorname{Vol}_{n}\left(\sum_{i=1}^{m}\left(\sum_{j=1}^{r_{i}} \lambda_{i j}\right) \mathcal{Q}^{(i)}\right) .
$$

Let

$$
\beta_{i}=\sum_{j=1}^{r_{i}} \lambda_{i, j} \text { for } i=1, \ldots, m,
$$

then the above expression becomes

$$
R\left(\beta_{1}, \ldots, \beta_{m}\right):=\operatorname{Vol}_{n}\left(\beta_{1} \mathcal{Q}^{(1)}+\beta_{2} \mathcal{Q}^{(2)}+\cdots+\beta_{m} \mathcal{Q}^{(m)}\right)
$$

which is a homogeneous polynomial of degree $n$ in the $\beta_{i}$ 's. Notice that by the multinomial expansion,

$$
\beta_{i}^{k}=\left(\sum_{j=1}^{r_{i}} \lambda_{i j}\right)^{k}=\sum_{t_{1}+\cdot+t_{r_{i}}=k} \frac{k !}{t_{1} ! \cdots t_{r_{i}} !} \prod_{j=1}^{r_{i}} \lambda_{i j}^{t_{j}} .
$$


Therefore a monomial in $\beta_{i}$ 's can be expanded as

$$
\beta_{1}^{k_{1}} \cdots \beta_{m}^{k_{m}}=\prod_{i=1}^{m}\left(\sum_{t_{i 1}+\cdots+t_{i r_{i}}=k_{i}} \frac{k_{i} !}{t_{i 1} ! \cdots t_{i r_{i}} !} \prod_{j=1}^{r_{i}} \lambda_{i j}^{t_{i j}}\right) .
$$

Clearly, such an expansion involves the monomial $\prod_{i=1}^{m} \prod_{j=1}^{r_{1}} \lambda_{1, j}$ precisely when $t_{i, j}=1$ for all $i=1, \ldots, m$ and $j=1, \ldots, r_{i}$ which yields $k_{i}=$ $\sum_{j=1}^{r_{i}} t_{i, j}=\sum_{j=1}^{r_{i}} 1=r_{i}$. Therefore in

$$
R\left(\beta_{1}, \ldots, \beta_{m}\right)=R\left(\left(\sum_{j=1}^{r_{1}} \lambda_{1, j}\right), \ldots,\left(\sum_{j=1}^{r_{m}} \lambda_{m, j}\right)\right),
$$

only the monomial $\beta_{1}^{r_{1}} \cdots \beta_{m}^{r_{m}}$ involves $\prod_{i=1}^{m} \prod_{j=1}^{r_{1}} \lambda_{i, j}$ in which it appears with coefficient $r_{1} ! \cdots r_{m}$ !

Proposition 17. For semi-mixed system $\mathcal{S}=\left(\mathcal{S}^{(1)}, r_{1} ; \mathcal{S}^{(2)}, r_{2} ; \cdots ; \mathcal{S}^{(m)}\right.$, $\left.r_{m}\right)$, the mixed volume $\mathcal{M}\left(\mathcal{Q}^{(1)}, r_{1} ; \mathcal{Q}^{(2)}, r_{2} ; \cdots ; \mathcal{Q}^{(m)}, r_{m}\right)$ is the coefficient of $\beta_{1}^{r_{1}} \cdots \beta_{m}^{r_{m}}$ in the polynomial

$$
\operatorname{Vol}_{n}\left(\beta_{1} \mathcal{Q}^{(1)}+\beta_{2} \mathcal{Q}^{(2)}+\cdots+\beta_{m} \mathcal{Q}^{(m)}\right)
$$

divided by $r_{1} ! \cdots r_{m} !$.

To utilize this observation in mixed volume computations, we slightly extend the framework of cells in which the mixed subdivision is defined for the Minkowski sum of polytopes in previous sections. With $\mathcal{S}^{(i)}$ being a finite subset of $\mathbb{R}^{n}$ for $i=1, \ldots, m$, and $m \leq n$, a cell of the $m$-tuple $\mathcal{S}=$ $\left(\mathcal{S}^{(1)}, \ldots, \mathcal{S}^{(m)}\right)$ is now an $m$-tuple $C=\left(C_{1}, \ldots, C_{m}\right)$ of nonempty subsets $C_{i} \subseteq \mathcal{S}^{(i)}$. With similar notations

$$
\begin{aligned}
\text { type } C & =\left(\operatorname{dim}\left(\operatorname{conv} C_{1}\right), \ldots, \operatorname{dim}\left(\operatorname{conv} C_{m}\right)\right) \\
\operatorname{conv} C & =\operatorname{conv} C_{1}+\cdots+\operatorname{conv} C_{m} \\
\operatorname{Vol}_{n} C & =\operatorname{Vol}_{n}(\operatorname{conv} C)
\end{aligned}
$$

we give the following:

Definition 18 (Fine semi-mixed subdivision). A fine semi-mixed subdivision of the $m$-tuple $\mathcal{S}=\left(\mathcal{S}^{(1)}, \ldots, \mathcal{S}^{(m)}\right)$ is a collection $\mathcal{D}$ of cells of $\mathcal{S}$ such that 
(a): $\operatorname{dim}(\operatorname{conv} C)=n$ for all $C \in \mathcal{D}$;

(b): For a distinct pair $A, B \in \mathcal{D}$, if $(\operatorname{conv} A) \cap(\operatorname{conv} B)$ is nonempty, then it is a common face of both;

(c): $\bigcup_{C \in \mathcal{D}} \operatorname{conv} C=\operatorname{conv} \mathcal{S}^{(1)}+\cdots+\operatorname{conv} \mathcal{S}^{(m)}$;

(d1): For each cell $C=\left(C_{1}, \ldots, C_{m}\right) \in \mathcal{D}, \sum_{i=1}^{m} \operatorname{dim}\left(\operatorname{conv} C_{i}\right)=n$

(d2): For distinct pair of cells $A=\left(A_{1}, \ldots, A_{m}\right), B=\left(B_{1}, \ldots, B_{m}\right) \in \mathcal{D}$,

$$
(\operatorname{conv} A) \cap(\operatorname{conv} B)=\sum_{i=1}^{m}\left(\operatorname{conv} A_{i} \cap \operatorname{conv} B_{i}\right)
$$

(e): For each cell $C=\left(C_{1}, \ldots, C_{m}\right) \in \mathcal{D}$, conv $C_{i}$ is a simplex of dimension $\# C_{i}-1$ for $i=1, \ldots, m$.

Notice that replacing all the $m$ 's by $n$ in the above yields exactly the same fine mixed subdivision in Definition 1, 2, and 3. Most importantly, the properties of fine mixed subdivisions proved in 92 can be preserved with minor adjustments. In particular, the scaling invariance of a fine semi-mixed subdivision remains valid:

Proposition 19. If $\mathcal{D}$ is a fine semi-mixed subdivision of the $m$-tuple $\mathcal{S}=$ $\left(\mathcal{S}^{(1)}, \ldots, \mathcal{S}^{(m)}\right)$, and $\boldsymbol{\beta}=\left(\beta_{1}, \ldots, \beta_{m}\right) \in\left(\mathbb{R}^{+}\right)^{m}$, the set

$$
\boldsymbol{\beta} \circ \mathcal{D}:=\left\{\boldsymbol{\beta} \circ C=\left(\beta_{1} C_{1}, \ldots, \beta_{m} C_{m}\right): C=\left(C_{1}, \ldots, C_{m}\right) \in \mathcal{D}\right\}
$$

forms a fine semi-mixed subdivision of $\boldsymbol{\beta} \circ \mathcal{S}=\left(\beta_{1} \mathcal{S}^{(1)}, \ldots, \beta_{m} \mathcal{S}^{(m)}\right)$.

Also, similar to 11 , the volume $\operatorname{Vol}_{n}\left(\beta_{1} \mathcal{Q}^{(1)}+\beta_{2} \mathcal{Q}^{(2)}+\cdots+\beta_{m} \mathcal{Q}^{(m)}\right)$ can be expressed in terms of volumes of cells of a fine semi-mixed subdivision:

Proposition 20. If $\mathcal{D}$ is a fine semi-mixed subdivision of the $m$-tuple $\mathcal{S}=$ $\left(\mathcal{S}^{(1)}, \ldots, \mathcal{S}^{(m)}\right)$, and $\boldsymbol{\beta}=\left(\beta_{1}, \ldots, \beta_{m}\right) \in\left(\mathbb{R}^{+}\right)^{m}$,

$$
\operatorname{Vol}_{n}\left(\beta_{1} \mathcal{Q}^{(1)}+\cdots+\beta_{m} \mathcal{Q}^{(m)}\right)=\sum_{C \in \mathcal{D}} \beta_{1}^{k_{1}^{C}} \cdots \beta_{m}^{k_{m}^{C}} \operatorname{Vol}_{n}(C)
$$

where $\left(k_{1}^{C}, \ldots, k_{m}^{C}\right)$ denotes the type of $C=\left(C_{1}, \ldots, C_{m}\right) \in \mathcal{D}$. 
Similar to $(6)$, in this semi-mixed context we define

$$
V(C)=\left[\begin{array}{lll}
V\left(C_{1}\right) & \cdots & V\left(C_{m}\right)
\end{array}\right]
$$

in which the block $V\left(C_{i}\right)$ will not appear if $\# C_{i}=1$. When $\mathcal{D}$ is a fine semimixed subdivision, similar to 10$)$, the volume of a cell $C=\left(C_{1}, \ldots, C_{m}\right) \in$ $\mathcal{D}$ can be computed via the formula

$$
\operatorname{Vol}_{n}(C)=\frac{1}{k_{1}^{C} ! \cdots k_{m}^{C} !}|\operatorname{det} V(C)|
$$

where $\left(k_{1}^{C}, \ldots, k_{m}^{C}\right)=\operatorname{type}(C)$. This yields the expression of the mixed volume of a semi-mixed system as a generalization of (12):

Proposition 21. Let $\mathcal{D}$ be a fine semi-mixed subdivision of the semi-mixed system $\mathcal{S}=\left(\mathcal{S}^{(1)}, r_{1} ; \cdots ; \mathcal{S}^{(m)}, r_{m}\right)$. The mixed volume $\mathcal{M}\left(\mathcal{Q}^{(1)}, r_{1} ; \cdots ; \mathcal{Q}^{(m)}\right.$, $\left.r_{m}\right)$ of this system is

$$
\mathcal{M}\left(\mathcal{Q}^{(1)}, r_{1} ; \mathcal{Q}^{(2)}, r_{2} ; \cdots ; \mathcal{Q}^{(m)}, r_{m}\right)=\sum_{\substack{C \in \mathcal{D} \\ \operatorname{type}(C)=\left(r_{1}, \ldots, r_{m}\right)}}|\operatorname{det} V(C)|
$$

with $V(C)$ as defined in 19 .

Moreover, similar to inducing a fine mixed subdivision for the fully mixed case $\mathcal{S}=\left(\mathcal{S}_{1}, \ldots, \mathcal{S}_{n}\right)$ by a generic lifting function as described in $\$ 5$, the same procedure can be followed almost line by line to construct a induced fine semi-mixed subdivision for a semi-mixed system $\mathcal{S}=\left(\mathcal{S}^{(1)}, r_{1} ; \cdots ; \mathcal{S}^{(m)}\right.$, $\left.r_{m}\right)$. Hence Proposition 16 can be generalized to the semi-mixed system:

Proposition 22 (Induced fine semi-mixed subdivision). Let $\boldsymbol{\omega}=$ $\left(\boldsymbol{\omega}_{1}, \ldots, \boldsymbol{\omega}_{m}\right)$ be a generic lifting function for the $m$-tuple $\mathcal{S}=\left(\mathcal{S}^{(1)}, \ldots, \mathcal{S}^{(m)}\right)$. Define

$$
\hat{\mathcal{S}}^{(i)}:=\left\{\left(\boldsymbol{a}, \boldsymbol{\omega}_{i}(\boldsymbol{a})\right): \boldsymbol{a} \in \mathcal{S}^{(i)}\right\},
$$

for each $i=1, \ldots, m$. Let $\hat{\mathcal{D}}_{\boldsymbol{\omega}}$ be the collection of all $\hat{C}=\left(\hat{C}_{1}, \ldots, \hat{C}_{m}\right)$ with $\hat{C}_{i} \subseteq \hat{\mathcal{S}}^{(i)}$ for each $i=1, \ldots$, m such that

1) $\operatorname{conv} \hat{C}_{i}$ is a face of conv $\hat{\mathcal{S}}_{i}$ for each $i=1, \ldots, m$;

2) The $m$ faces conv $\hat{C}_{i}$ of conv $\hat{\mathcal{S}}^{(i)}$ for $i=1, \ldots, m$ respectively share a common inner normal of the form $\hat{\boldsymbol{\alpha}}=\left(\alpha_{1}, \ldots, \alpha_{n}, 1\right)$; and

3) $\operatorname{dim}\left(\operatorname{conv} \hat{C}_{1}+\cdots+\operatorname{conv} \hat{C}_{m}\right)=n$. 
Then the projection of $\hat{\mathcal{D}}_{\boldsymbol{\omega}}$

$$
\left.\mathcal{D}_{\boldsymbol{\omega}}=\left\{\left(\pi\left(\hat{C}_{1}\right), \ldots, \pi\left(\hat{C}_{m}\right)\right)\right):\left(\hat{C}_{1}, \ldots, \hat{C}_{m}\right) \in \hat{\mathcal{D}}_{\boldsymbol{\omega}}\right\}
$$

form a fine semi-mixed subdivision of $\mathcal{S}=\left(\mathcal{S}^{(1)}, \ldots, \mathcal{S}^{(m)}\right)$, and it is called the fine semi-mixed subdivision induced by the lifting function $\omega$.

\section{Enumeration of mixed cells}

The crux of our strategy for calculating mixed volume $\mathcal{M}\left(\mathcal{Q}^{(1)}, r_{1} ; \cdots\right.$; $\left.\mathcal{Q}^{(m)}, r_{m}\right)$ of a semi-mixed system $\mathcal{S}=\left(\mathcal{S}^{(1)}, r_{1} ; \cdots ; \mathcal{S}^{(m)}, r_{m}\right)$, where $\mathcal{Q}^{(i)}=$ conv $\mathcal{S}^{(i)}$, is the identification of the mixed cells of type $\left(r_{1}, \ldots, r_{m}\right)$ in the fine semi-mixed subdivision $\mathcal{D}_{\boldsymbol{\omega}}$ induced by the generic lifting function $\boldsymbol{\omega}:=\left(\boldsymbol{\omega}_{1}, \ldots, \boldsymbol{\omega}_{m}\right)$ as provided by Proposition 22, Algebraically, a mixed cell $C \in \mathcal{D}_{\boldsymbol{\omega}}$ of type $\left(r_{1}, \ldots, r_{m}\right)$, is an $m$-tuple

$$
C=\left(\left\{\boldsymbol{a}_{0}^{(1)}, \ldots, \boldsymbol{a}_{r_{1}}^{(1)}\right\}, \ldots,\left\{\boldsymbol{a}_{0}^{(m)}, \ldots, \boldsymbol{a}_{r_{m}}^{(m)}\right\}\right)
$$

with $\boldsymbol{a}_{j}^{(i)} \in \mathcal{S}^{(i)}$ for $i=1, \ldots, m$ and $j=0, \ldots, r_{i}$ for which there exists an $\boldsymbol{\alpha}=\left(\alpha_{1}, \ldots, \alpha_{n}\right) \in \mathbb{R}^{n}$ such that for each $i=1, \ldots, m$,

$$
\begin{array}{ll}
\left\langle\hat{\boldsymbol{a}}_{0}^{(i)}, \hat{\boldsymbol{\alpha}}\right\rangle=\left\langle\hat{\boldsymbol{a}}_{j}^{(i)}, \hat{\boldsymbol{\alpha}}\right\rangle & \text { for } j=0, \ldots, r_{i} \\
\left\langle\hat{\boldsymbol{a}}_{0}^{(i)}, \hat{\boldsymbol{\alpha}}\right\rangle \leq\langle\hat{\boldsymbol{a}}, \hat{\boldsymbol{\alpha}}\rangle & \text { for } \boldsymbol{a} \in \mathcal{S}^{(i)}
\end{array}
$$

where $\hat{\boldsymbol{\alpha}}:=(\boldsymbol{\alpha}, 1)^{\top}=\left(\alpha_{1}, \ldots, \alpha_{n}, 1\right)^{\top}$. This algebraic description of mixed cells in $\mathcal{D}_{\boldsymbol{\omega}}$ of type $\left(r_{1}, \ldots, r_{m}\right)$ is the basis on which mixed cell enumeration algorithms are developed: the problem is now reduced to a search problem for choices of $r_{1}, \ldots, r_{m}$ points from $\mathcal{S}^{(1)}, \ldots, \mathcal{S}^{(m)}$ respectively for which the system of inequalities (22) has a solution. There are several approaches to carrying out such search. One of the most efficient class of algorithms is based on the idea of systematic "extension of subfaces".

This scheme first locates the $r_{1}$-dimensional lower faces of $\hat{\mathcal{Q}}^{(1)}$, that is, the faces of $\hat{\mathcal{Q}}_{1}$ that has an inner normal with 1 being the last coordinate. These faces are known as level-1 "subfaces". Namely, one first finds all possible choices $\left\{\boldsymbol{a}_{0}^{(1)}, \ldots, \boldsymbol{a}_{r_{1}}^{(1)}\right\}$ of $r_{1}+1$ points in $\mathcal{S}^{(1)}$ for which there exists an $\hat{\boldsymbol{\alpha}}=\left(\alpha_{1}, \ldots, \alpha_{n}, 1\right)^{\top} \in \mathbb{R}^{n+1}$ such that

$$
\begin{array}{ll}
\left\langle\hat{\boldsymbol{a}}_{0}^{(1)}, \hat{\boldsymbol{\alpha}}\right\rangle=\left\langle\hat{\boldsymbol{a}}_{j}^{(1)}, \hat{\boldsymbol{\alpha}}\right\rangle & \text { for } j=0, \ldots, r_{1} \\
\left\langle\hat{\boldsymbol{a}}_{0}^{(i)}, \hat{\boldsymbol{\alpha}}\right\rangle \leq\langle\hat{\boldsymbol{a}}, \hat{\boldsymbol{\alpha}}\rangle & \text { for } \boldsymbol{a} \in \mathcal{S}^{(1)} .
\end{array}
$$


The testing for the existence of solutions for the above system of inequalities is generally known as the "Phase I", or feasibility, problem in Linear Programming.

Then for each of these level-1 subfaces, systematic attempts are made to "extend" it by finding a $r_{2}$-dimensional lower face of $\hat{\mathcal{Q}}^{(2)}$ so that the two lower faces (of $\hat{\mathcal{Q}}^{1)}$ and $\hat{\mathcal{Q}}^{(2)}$ respectively) can share a common inner normal of the form $\hat{\boldsymbol{\alpha}}=\left(\alpha_{1}, \ldots, \alpha_{n}, 1\right)^{\top}$ of both $\hat{\mathcal{Q}}^{(1)}$ and $\hat{\mathcal{Q}}^{(2)}$. That is, fixing a level-1 subface defined by $\boldsymbol{a}_{0}^{(1)}, \ldots, \boldsymbol{a}_{r_{1}}^{(1)}$ one attempts to find a $r_{2^{-}}$ dimensional lower face $\operatorname{conv}\left\{\hat{\boldsymbol{a}}_{0}^{(2)}, \ldots, \hat{\boldsymbol{a}}_{r_{2}}^{(2)}\right\}$ of $\hat{\mathcal{Q}}^{(2)}$ with $\hat{\boldsymbol{a}}_{j}^{(2)} \in \hat{\mathcal{S}}^{(2)}$ for each $j=0, \ldots, r_{2}$ such that the system 23 and the system

$$
\begin{array}{ll}
\left\langle\hat{\boldsymbol{a}}_{0}^{(2)}, \hat{\boldsymbol{\alpha}}\right\rangle=\left\langle\hat{\boldsymbol{a}}_{j}^{(2)}, \hat{\boldsymbol{\alpha}}\right\rangle & \text { for } j=0, \ldots, r_{2} \\
\left\langle\hat{\boldsymbol{a}}_{0}^{(i)}, \hat{\boldsymbol{\alpha}}\right\rangle \leq\langle\hat{\boldsymbol{a}}, \hat{\boldsymbol{\alpha}}\rangle & \text { for } \boldsymbol{a} \in \mathcal{S}^{(2)}
\end{array}
$$

have a common solution $\hat{\boldsymbol{\alpha}}=\left(\alpha_{1}, \ldots, \alpha_{n}, 1\right)^{\top} \in \mathbb{R}^{n+1}$. The testing for the existence of a common solution is, again, a classical Phase I problem in Linear Programming. Note that this enlarged system of (23) and (24) may not have a solution for all choices of $\left\{\hat{\boldsymbol{a}}_{0}^{(2)}, \ldots, \hat{\boldsymbol{a}}_{r_{2}}^{(2)}\right\} \subseteq \hat{\mathcal{S}}^{(2)}$. In this case, no lower face of $\hat{\mathcal{Q}}^{(2)}$ can extend the chosen level-1 subface, and the extension attempt will be restarted on some other level-1 subfaces. On the other hand, the possible $r_{2}$-dimensional lower faces of $\hat{\mathcal{Q}}^{(2)}$ that can extend the chosen level-1 subface may not be unique, and each of such possible extension is known as a level-2 subface. They will be extended further individually into possible level3 subfaces. This process may continue until one reaches level- $m$ subfaces, each of which is an $m$-tuple of lower faces of $\hat{\mathcal{Q}}^{(1)}, \ldots, \hat{\mathcal{Q}}^{(m)}$ with dimensions $r_{1}, \ldots, r_{m}$ respectively that share a common inner normal vector of the form $\hat{\boldsymbol{\alpha}}=\left(\alpha_{1}, \ldots, \alpha_{n}, 1\right)^{\top}$. This is precisely a cell in $\hat{\mathcal{D}}_{\boldsymbol{\omega}}$ whose projection (by erasing the last coordinate) is a fine semi-mixed cell of type $\left(r_{1}, \ldots, r_{m}\right)$ in $\mathcal{D}_{\boldsymbol{\omega}}$ as given in Proposition 22. Once all such mixed cells of type $\left(r_{1}, \ldots, r_{m}\right)$ have been found, the mixed volume $\mathcal{M}\left(\mathcal{Q}^{(1)}, r_{1} ; \cdots ; \mathcal{Q}^{(m)}, r_{m}\right)$ of a semi-mixed system $\mathcal{S}=\left(\mathcal{S}^{(1)}, r_{1} ; \cdots ; \mathcal{S}^{(m)}, r_{m}\right)$ can be computed via formula 20 .

This scheme of "extension of subfaces" has been developed into a class of efficient, robust, and highly parallel algorithms which have been implemented in software packages such as [4, 11, 12, 19, 22, 26].

\section{References}

[1] D. N. Bernshtein, The number of roots of a system of equations. Functional Analysis and its Applications, 9(3):183-185, 1975. 
[2] U. Betke, Mixed volumes of polytopes. Archiv der Mathematik, 58(4): 388-391, Apr. 1992.

[3] J. Canny and J. M. Rojas, An optimal condition for determining the exact number of roots of a polynomial system. In "Proceedings of the 1991 International Symposium on Symbolic and Algebraic Computation", ISSAC '91, pages 96-102, New York, NY, USA, 1991. ACM.

[4] T. R. Chen, T. L. Lee and T. Y. Li, Mixed volume computation in parallel. Taiwanese Journal of Mathematics, 18(1):93-114, 2014.

[5] T. R. Chen, T. L. Lee and T. Y. Li, Hom4PS-3: A parallel numerical solver for systems of polynomial equations based on polyhedral homotopy continuation method. Journal of Symbolic Computation, Sumitted.

[6] D. A. Cox, J. B. Little and D. O'shea, Using Algebraic Geometry. 2005.

[7] M. Dyer, P. Gritzmann and A. Hufnagel, On the complexity of computing mixed volumes. SIAM Journal on Computing, 27(2):356-400, Apr. 1998.

[8] I. Z. Emiris and J. F. Canny, Efficient incremental algorithms for the sparse resultant and the mixed volume. Journal of Symbolic Computation, 20(2):117-149, 1995.

[9] I. Z. Emiris and J. Verscheide, How to count efficiently all affine roots of a polynomial system. Discrete Applied Mathematics, 93(1):21-32, Apr. 1999.

[10] G. Ewald, Minkowski sum and mixed volume. In "Combinatorial Convexity and Algebraic Geometry", number 168 in Graduate Texts in Mathematics, pages 103-141. Springer New York, Jan. 1996.

[11] T. Gao and T. Y. Li, Mixed volume computation via linear programming. Taiwanese Journal of Mathematics, 4(4):599-619, Jan. 2000.

[12] T. Gao and T.-Y. Li, Mixed volume computation for semi-mixed systems. Discrete \& Computational Geometry, 29(2):257-277, Jan. 2003.

[13] H. Groemer, Minkowski addition and mixed volumes. Geometriae Dedicata, 6(2):141-163, Aug. 1977.

[14] W. Gubler, A guide to tropicalizations. In "Algebraic and combinatorial aspects of tropical geometry", pages 125-189, 2013.

[15] T. Gunji, S. Kim, M. Kojima, A. Takeda, K. Fujisawa and T. Mizutani, PHoMa polyhedral homotopy continuation method for polynomial systems. Computing, 73(1):57-77, 2004. 
[16] B. Huber and B. Sturmfels, A polyhedral method for solving sparse polynomial systems. Mathematics of computation, 64(212):1541-1555, 1995.

[17] I. Itenberg, G. Mikhalkin and E. I. Shustin, Tropical Algebraic Geometry. Springer Science \& Business Media, May 2009.

[18] A. Khovanskii, Newton polyhedra and the genus of complete intersections. Functional Analysis and Its Applications, 12(1):38-46, 1978.

[19] T. L. Lee and T. Y. Li, Mixed volume computation in solving polynomial systems. Contemp. Math, 556:97-112, 2011.

[20] T. L. Lee, T. Y. Li and C. H. Tsai, HOM4PS-2.0: a software package for solving polynomial systems by the polyhedral homotopy continuation method. Computing, 83(2):109-133, 2008.

[21] T. Y. Li, Numerical solution of polynomial systems by homotopy continuation methods. In "Handbook of Numerical Analysis" (P. G. Ciarlet ed.), volume 11, pages 209-304. North-Holland, 2003.

[22] T. Y. Li and X. Li, Finding mixed cells in the mixed volume computation. Foundations of Computational Mathematics, 1(2):161-181, Jan. 2001.

[23] T. Y. Li and X. Wang, The BKK root count in $\mathbb{C}^{n}$. Mathematics of Computation of the American Mathematical Society, 65(216):1477$1484,1996$.

[24] T. Michiels and J. Verschelde, Enumerating regular mixed-cell configurations. Technical report, Discrete \& Computational Geometry, 1997.

[25] H. Minkowski, Theorie der konvexen körper, insbesondere begründung ihres oberflächenbegriffs. Gesammelte Abhandlungen von Hermann Minkowski, 2:131-229, 1911.

[26] T. Mizutani and A. Takeda, DEMiCs: A software package for computing the mixed volume via dynamic enumeration of all mixed cells. In "Software for Algebraic Geometry" (M. Stillman, J. Verschelde and N. Takayama eds.), number 148 in The IMA Volumes in Mathematics and its Applications, page 59-79. Springer, Jan. 2008.

[27] J. M. Rojas, Toric intersection theory for affine root counting. Journal of Pure and Applied Algebra, 136(1):67-100, Mar. 1999.

[28] J. M. Rojas, Why polyhedra matter in non-linear equation solving. Contemporary Mathematics, 334:293-320, 2003. 
[29] J. Verschelde, Algorithm 795: PHCpack: A general-purpose solver for polynomial systems by homotopy continuation. ACM Transactions on Mathematical Software (TOMS), 25(2):251-276, 1999.

[30] C. W. Wampler, A. P. Morgan and A. J. Sommese, Complete solution of the nine-point path synthesis problem for four-bar linkages. Journal of Mechanical Design, 114:153, 1992.

Department of Mathematics, Michigan State University

EAst LANSInG, MI 48823, USA

E-mail address: chentia1@msu.edu

Department of Mathematics, Michigan State University

EAst LANSInG, MI 48823, USA

E-mail address: li@math.msu.edu

Department of Mathematics and Statistics

University of Arkansas at Little Rock

LitTle Rock, AR 72204, USA

E-mail address: xxwang@ualr.edu

Received SEPtember 26, 2014

ACCePted March 11, 2015 